\title{
Towards the Willmore conjecture
}

\author{
Peter Topping \\ University of Warwick, Mathematics Institute, Coventry CV4 7AL, UK \\ (topping@maths.warwick.ac.uk; http://www.maths.warwick.ac.uk/ topping/)
}

Received April 26, 1999 / Accepted January 14, 2000 /

Published online June 28, 2000 - (C) Springer-Verlag

\begin{abstract}
We develop a variety of approaches, mainly using integral geometry, to proving that the integral of the square of the mean curvature of a torus immersed in $\mathbb{R}^{3}$ must always take a value no less than $2 \pi^{2}$. Our partial results, phrased mainly within the $S^{3}$-formulation of the problem, are typically strongest when the Gauss curvature can be controlled in terms of extrinsic curvatures or when the torus enjoys further properties related to its distribution within the ambient space (see Sect. 3). Corollaries include a recent result of Ros [20] confirming the Willmore conjecture for surfaces invariant under the antipodal map, and a strengthening of the expected results for flat tori.

The value $2 \pi^{2}$ arises in this work in a number of different ways - as the volume (or renormalised volume) of $S^{3}, S O(3)$ or $G_{2,4}$, and in terms of the length of shortest nontrivial loops in subgroups of $S O(4)$.
\end{abstract}

Mathematics Subject Classification (1991):49Q10, 53C65

\section{Statement of the problem, and opening remarks}

Let $v: \mathcal{M} \rightarrow \mathbb{R}^{3}$ be a smooth immersion of a compact orientable two dimensional surface. Giving $\mathcal{M}$ the metric induced by $v$, and considering the mean curvature $\hat{H}: \mathcal{M} \rightarrow \mathbb{R}$ defined to be the mean of the principal curvatures $\hat{\kappa}_{1}$ and $\hat{\kappa}_{2}$ at each point (which is defined up to a choice of sign) we may consider the Willmore energy of the surface, defined to be

$$
W=W(\mathcal{M})=\int_{\mathcal{M}} \hat{H}^{2} .
$$


Smooth surfaces which are critical points of the functional $W$ with respect to normal variations have been studied since the first quarter of the previous century and the works of Blaschke [4], Schadow and Thomsen [22]; nowadays they are commonly referred to as Willmore surfaces having been reinvented and popularised by Willmore in the past few decades.

In this work we shall be seeking the infimum of $W$ over all immersions with fixed genus. To begin with, it is not hard to see that

$$
W \geq 4 \pi,
$$

for immersed surfaces of any genus, with equality precisely for round embedded spheres. Indeed, if we write $\mathcal{M}_{+}=\{x \in \mathcal{M} \mid \hat{K}(x) \geq 0\}$, where the Gauss curvature $\hat{K}: \mathcal{M} \rightarrow \mathbb{R}$ is the product $\hat{\kappa}_{1} \hat{\kappa}_{2}$ of the principal curvatures, then we have

$$
W \geq \int_{\mathcal{M}_{+}} \hat{H}^{2} \geq \int_{\mathcal{M}_{+}} \hat{K} \geq 4 \pi
$$

where we have used the fact that $\hat{H}^{2}-\hat{K}=\frac{1}{4}\left(\hat{\kappa}_{1}-\hat{\kappa}_{2}\right)^{2} \geq 0$, and where the final inequality follows because $\hat{K}$ is the Jacobian of the Gauss map $\hat{\mathcal{G}}: \mathcal{M} \rightarrow S^{2} \hookrightarrow \mathbb{R}^{3}$ which maps $\mathcal{M}_{+}$onto the target which has area $4 \pi$. In the case of equality, we clearly have $\hat{H}^{2}-\hat{K} \equiv 0$, and the only totally umbilic compact surfaces in $\mathbb{R}^{3}$ are round spheres, for which we clearly have equality.

However, for surfaces of genus greater than zero we expect a bound somewhat stronger than (1). In particular, calculations of $W$ for tori with various symmetries led Willmore to the following belief.

Conjecture 1 Given any smooth immersed torus in $\mathbb{R}^{3}$, the Willmore energy should be bound by the inequality

$$
W \geq 2 \pi^{2} .
$$

Equality is achieved in (3) for the embedding of the torus $S^{1} \times S^{1}$ given by

$$
(\theta, \varphi) \rightarrow((\sqrt{2}+\cos \varphi) \cos \theta,(\sqrt{2}+\cos \varphi) \sin \theta, \sin \varphi) .
$$

It is no fluke (see the next section) that this surface is a stereographic projection into $\mathbb{R}^{3}$ of the Clifford torus. Despite the simplicity of the statement of this conjecture, it remains open after much attention since its formulation in 1965. However, we have no deep obstruction to the existence of an elementary proof. For background on this conjecture from the perspective of Willmore himself, we refer the reader to [25, Chapter 7].

In $[24$, Sect. 0.6] (see inequality (22) of that paper) we proved the following sharp improvement of (2) with a view to proving diameter bounds for surfaces. 
Lemma 1 Suppose $v: \mathcal{M} \rightarrow \mathbb{R}^{3}$ is an immersed surface which covers the point $x \in \mathbb{R}^{3}$ a total of $k$ times. Then writing $\mathcal{M}_{\rho}=\{y \in \mathcal{M}:|v(y)-x|<$ $\rho$ \} for $\rho>0$, we have

$$
k \pi \leq \rho^{-2} \operatorname{Area}\left(\mathcal{M}_{\rho}\right)+\frac{1}{4} W\left(\mathcal{M}_{\rho}\right) .
$$

In the limit $\rho \rightarrow \infty$, this local estimate becomes the global estimate

$$
4 k \pi \leq W(\mathcal{M})
$$

first proved by $\mathrm{Li}$ and Yau [11]. In particular, any surface with a selfintersection must have $W \geq 8 \pi$. Since we already have a torus, given by (4), with $W=2 \pi^{2}<8 \pi$, we see that it suffices to prove the Willmore conjecture for embedded tori. Moreover, a result of Rosenberg and Langevin [14] tells us that any knotted torus embedded in $\mathbb{R}^{3}$ must have total curvature of at least $16 \pi$ (where the total curvature is defined to be the integral of $|\hat{K}|$ ) and in this case

$$
W \geq \int_{\mathcal{M}_{+}} \hat{H}^{2} \geq \int_{\mathcal{M}_{+}} \hat{K}=\frac{1}{2} \int_{\mathcal{M}}|\hat{K}| \geq 8 \pi,
$$

using the Gauss-Bonnet formula. Therefore, it suffices to prove the Willmore conjecture for embedded tori which are isotopic to the torus given by (4).

In fact, whilst the map (4) is expected to minimise $W$ for tori, there are also candidates for minimisers when the genus is greater than one. These are derived from certain minimal surfaces in $S^{3}$ (see the next section) which were constructed by Lawson [15] (see Kusner [12]). Both Pinkall and Kusner [12] have calculated, independently, that the Willmore energy for these surfaces is always less than $8 \pi$, and hence we may also assume embeddedness for the higher genus analogues to Willmore's conjecture. It is worth remarking that the weaker statement that the infimum of $W$ is no more than $8 \pi$ for any genus is rather easier to see, using a variant of an idea of Nadirashvili: taking two concentric round spheres with almost identical radii, we may 'rivet' them together by cutting out small adjacent discs from each, and replacing them by a slightly deformed catenoid (which adds an arbitrarily small amount to $W$ ). After $n+1$ rivets, in different places, we are left with a genus $n$ surface with $W$ arbitrarily close to $8 \pi$.

The existence of a torus attaining the minimum value of $W$ over all tori has been established by Leon Simon [21]; this work has since been extended to cover any genus by Kusner [13]. The existence of a minimiser opened up the possibility of a direct assault on the Euler-Lagrange equation

$$
-\Delta \hat{H}=2\left(\hat{H}^{2}-\hat{K}\right) \hat{H},
$$

satisfied by Willmore surfaces, in order to prove the Willmore conjecture. Unfortunately, (6) does not appear to encode enough information; it seems 
crucial to use the minimality, and not just the criticality of the torus, since the class of all Willmore tori turns out to be rather large and difficult to control (see [19] and [1]). One consequence of Simon's work is that the infimum of $W$ over all tori is strictly greater than $4 \pi$. We used this fact in [24] to prove the existence of singularities in the solutions of certain nonlinear evolution equations.

Finally, let us return to our considerations of the Gauss map to make two philosophical remarks concerning why sharp estimates for $W$ are simple for spheres, and difficult for tori. To begin with, we observe that a first attempt at (2) for surfaces of genus $g$ would be

$$
W=\int_{\mathcal{M}} \hat{H}^{2} \geq \int_{\mathcal{M}} \hat{K}=4 \pi(1-g),
$$

using the Gauss-Bonnet formula, which yields nothing for genus one (or larger) but gives the sharp estimate for genus zero. We are being helped by the fact that the Gauss map for spheres, being of degree one, is forced by topology to map to an area of at least $4 \pi$, and we are being hindered by the fact that the Gauss map for tori, being of degree zero, has no such topological restriction. Next, we record that the Willmore energy is related to the harmonic energy

$$
E(\hat{\mathcal{G}}):=\frac{1}{2} \int_{\mathcal{M}}|\nabla \hat{\mathcal{G}}|^{2}=\frac{1}{2} \int_{\mathcal{M}}\left(\hat{\kappa}_{1}^{2}+\hat{\kappa}_{2}^{2}\right)
$$

of the Gauss map according to

$$
W=\frac{1}{2}\left(E(\hat{\mathcal{G}})+\int_{\mathcal{M}} \hat{K}\right) .
$$

Since the harmonic energy of a map is always greater than the (geometric) area of its image, with equality precisely when the map is conformal (see [23] or [10]) we find that

$$
W \geq \frac{1}{2}\left(\int_{\mathcal{M}}|\hat{K}|+\int_{\mathcal{M}} \hat{K}\right)=\int_{\mathcal{M}_{+}} \hat{K}
$$

as in (2), with equality precisely when the Gauss map is conformal. We are therefore being helped by the fact that the Gauss map for a sphere may be conformal (when the sphere is round) allowing a sharp estimate, but we are hindered by the fact that the Gauss map for a torus cannot be conformal since it is of degree zero.

In what follows, we are repeatedly seeking, with varying degrees of success, to generalise the notion of Gauss map for tori in order to make it both topologically nontrivial, and conformal, whilst preserving its connection to the Willmore energy. 


\section{The $S^{3}$ framework}

During most of this work, we will be considering an equivalent formulation of the Willmore conjecture in which the ambient space is $S^{3}$ rather than $\mathbb{R}^{3}$. Given a smooth immersion $u: \mathcal{M} \rightarrow S^{3} \hookrightarrow \mathbb{R}^{4}$ of a compact orientable two dimensional surface into the standard 3-sphere, we may again allow $\mathcal{M}$ to take the metric induced by the ambient space, and if we write the principal curvatures as $\kappa_{1}$ and $\kappa_{2}$, we now have the mean and Gauss curvatures given by $H=\frac{1}{2}\left(\kappa_{1}+\kappa_{2}\right)$ and $K=1+\kappa_{1} \kappa_{2}$ respectively. Let us record now that one representation of the Gauss map for the surface in $S^{3}$ is the polar map

$$
u^{*}: \mathcal{M} \rightarrow S^{3} \hookrightarrow \mathbb{R}^{4},
$$

defined to be one of the two continuous maps which are orthogonal, at each point, both to the surface, and to the image point $u$ in $S^{3} \hookrightarrow \mathbb{R}^{4}$. (Our convention for choosing which of these maps to take, once we have an orientation on the surface and on the ambient $\mathbb{R}^{4}$, is that $\left\{u, u^{*}, e, f\right\}$ should be a positive basis for $\mathbb{R}^{4}$ when $\{e, f\}$ is a positive basis for the tangent space.) However, we shall normally reserve the term 'Gauss map' to refer to the map

$$
\mathcal{G}: \mathcal{M} \rightarrow G_{2,4}
$$

into the Grassmannian of oriented 2-planes in $\mathbb{R}^{4}$, which assigns, to each point on the surface, the oriented tangent plane to the surface.

We wish to compare the curvatures of a surface in $S^{3}$ and its image in $\mathbb{R}^{3}$ under stereographic projection $\varphi=\varphi_{e}: S^{3} \rightarrow \mathbb{R}^{3}$ sending the point $e \in S^{3} \hookrightarrow \mathbb{R}^{4}$ to the origin. In what follows, we will always have $\varphi \circ u=v$, and $x$ and $y$ will be local isothermal coordinates on $\mathcal{M}$ induced by $u$ or $v$ (note that $\varphi$ is conformal); we denote by $\hat{\rho}: \mathcal{M} \rightarrow \mathbb{R}$ and $\rho: \mathcal{M} \rightarrow \mathbb{R}$ the scaling factors which make $\hat{\gamma}=\hat{\rho}^{2}\left(d x^{2}+d y^{2}\right)$ and $\gamma=\rho^{2}\left(d x^{2}+d y^{2}\right)$ the metrics on $\mathcal{M}$ induced from $v$ and $u$ respectively. We also require the scaling factor $\sigma: \mathbb{R}^{3} \rightarrow \mathbb{R}$ defined by $\sigma(\mathbf{x})=\frac{2}{1+|\mathbf{x}|^{2}}$ which makes $\sigma^{2}\left(d x^{2}+d y^{2}+d z^{2}\right)$ the pull-back under $\varphi^{-1}$ of the standard metric on $S^{3}$. Clearly then, we have $\rho(x)=\sigma(v(x)) \hat{\rho}(x)$.

We may now compare the principal curvatures $\hat{\kappa}_{1}, \hat{\kappa}_{2}: \mathcal{M} \rightarrow \mathbb{R}$ in $\mathbb{R}^{3}$ and $\kappa_{1}, \kappa_{2}: \mathcal{M} \rightarrow \mathbb{R}$ in $S^{3}$. Assuming we have chosen a compatible sign for each set - i.e. we have chosen signs for the maps $\hat{\mathcal{G}}$ and $u^{*}$ so that $d \varphi_{u(x)}\left(u^{*}(x)\right)=\sigma^{-1}(v(x)) \hat{\mathcal{G}}(x)-$ a calculation shows that

$$
\kappa_{i}(x) \sigma(v(x))=\hat{\kappa}_{i}(x)-\langle v(x), \hat{\mathcal{G}}(x)\rangle \sigma(v(x))=\hat{\kappa}_{i}(x)+\left\langle e, u^{*}(x)\right\rangle,
$$

for $i=1,2$. In particular, we see that

$$
\left(\hat{\kappa}_{1}-\hat{\kappa}_{2}\right)^{2} \hat{\rho}^{2}=\left(\kappa_{1}-\kappa_{2}\right)^{2} \rho^{2},
$$


and hence, since

$$
H^{2}+1-K=\left(\kappa_{1}-\kappa_{2}\right)^{2} \geq 0
$$

we have

$$
\begin{aligned}
\int_{\mathcal{M}}\left(\hat{H}^{2}-\hat{K}\right) \hat{\rho}^{2} d x \wedge d y & =4 \int_{\mathcal{M}}\left(\hat{\kappa}_{1}-\hat{\kappa}_{2}\right)^{2} \hat{\rho}^{2} d x \wedge d y \\
& =4 \int_{\mathcal{M}}\left(\kappa_{1}-\kappa_{2}\right)^{2} \rho^{2} d x \wedge d y \\
& =\int_{\mathcal{M}}\left(H^{2}-K+1\right) \rho^{2} d x \wedge d y .
\end{aligned}
$$

Applying the Gauss-Bonnet formula then yields the formula for the Willmore energy of a surface once projected into $S^{3}$ :

$$
W=\int_{(\mathcal{M}, \hat{\gamma})} \hat{H}^{2}=\int_{(\mathcal{M}, \gamma)}\left(1+H^{2}\right) .
$$

A crucial property of the Willmore energy is its invariance under conformal transformations of the ambient space - a fact which may be found in the book of Blaschke [4]. Now we have formulated the Willmore energy in both $\mathbb{R}^{3}$ and $S^{3}$, this invariance is elementary to prove. Conformal transformations in these spaces are precisely the Möbius transformations, which may always be written as the composition of an isometry in $S^{3}$ (which clearly leaves $W$ unchanged) and a homothety. Homotheties in $S^{3}$ correspond to simple dilations in $\mathbb{R}^{3}$ after an appropriate stereographic projection, and dilations leave $W$ unchanged since the area scales quadratically whilst $\hat{H}^{2}$ scales inverse-quadratically. Of course, even the 'integrands' in (7) are invariant under the conformal transformations.

At various stages of this work we will have cause to consider the family of flat tori foliating $S^{3} \hookrightarrow \mathbb{C}^{2}$ given by

$$
\mathbb{T}_{r}:=\left\{\left(z_{1}, z_{2}\right) \in \mathbb{C}^{2}:\left|z_{1}\right|=r,\left|z_{2}\right|=\sqrt{1-r^{2}}\right\},
$$

for each $r \in(0,1)$, terminating at each end with great circles $\mathbb{T}_{0}$ and $\mathbb{T}_{1}$. The torus $\mathbb{T}_{\frac{1}{\sqrt{2}}}$ is then the Clifford torus - a minimal embedded torus in $S^{3}$ (and the only such torus according to a conjecture of Lawson - see [26, Problem 97]). Since the Clifford torus has $H \equiv 0$ and area $2 \pi^{2}$, we see immediately from (9) that its Willmore energy is $2 \pi^{2}$; it is precisely this torus, after stereographic projection, which we saw in (4), and should (according to the Willmore conjecture) minimise $W$ along with all of its images under conformal transformations of the ambient $S^{3}$.

Armed with the conformal invariance of $W$, we may describe a first partial result to the Willmore Conjecture, due to Li and Yau [11]. Given an 
immersed torus, the Uniformisation Theorem tells us that we may see it as a conformal immersion $u: \mathbb{T} \rightarrow S^{3}$ where $\mathbb{T}=\mathbb{R}^{2} / \Gamma$ for some lattice $\Gamma$ generated by $(1,0)$ and $(a, b)$ with $0 \leq a \leq \frac{1}{2}$ and $b \geq \sqrt{1-a^{2}}$. Following Szegő (see [11]) we may transform the ambient $S^{3}$ conformally so that the centre of mass of the torus ends up at the origin, in the sense that

$$
\int_{\mathbb{T}} u=0 \in \mathbb{R}^{4}
$$

where the integral is performed with respect to the flat metric $\left(d x^{2}+d y^{2}\right)$ on $\mathbb{T}$ naturally inherited from $\mathbb{R}^{2}$. We may then calculate

$$
\begin{aligned}
W=\int_{\mathbb{T}}\left(1+H^{2}\right) \rho^{2} \geq \int_{\mathbb{T}} \rho^{2} & =\sum_{i=1}^{4} \int_{\mathbb{T}} \frac{1}{2}\left|\nabla u^{i}\right|^{2} \geq \frac{1}{2} \lambda_{1}(\mathbb{T}) \sum_{i=1}^{4} \int_{\mathbb{T}}\left|u^{i}\right|^{2} \\
& =\frac{1}{2} \lambda_{1}(\mathbb{T}) \operatorname{Area}(\mathbb{T}) .
\end{aligned}
$$

where $\lambda_{1}(\mathbb{T})$ is the first eigenvalue of the Laplacian, and we have used the fact that $\sum_{i=1}^{4}\left|u^{i}\right|^{2}=1$. Since we know $\lambda_{1}(\mathbb{T})$ to be $\frac{4 \pi^{2}}{b^{2}}$ (see [3]) we find that

$$
W \geq \frac{2 \pi^{2}}{b},
$$

and hence that the Willmore conjecture holds for any torus whose conformal type satisfies $b \leq 1$; in particular it holds for any conformally square torus.

$\mathrm{Li}$ and Yau's original treatment of this result (using this method) was in terms of 'conformal volume' which we will not require during this article. The set of conformal structures covered by these methods was subsequently extended by Montiel and Ros [18].

\section{Survey of results}

Our first result, which will be a crude corollary of a more general estimate, addresses the case of flat tori. The Willmore conjecture has, in fact, been solved in this case by Chen [7] and improved in terms of restrictions on the conformal type of the torus by Li and Yau [11]. In contrast, our improvement is to the functional which may be estimated.

Theorem 1 Given an immersed torus in $S^{3}$ for which $K \equiv 0$, we have the estimate

$$
W=\int_{\mathcal{M}}\left(1+H^{2}\right) \geq \int_{\mathcal{M}} \sqrt{1+H^{2}} \geq 2 \pi^{2} .
$$


Of course the first inequality follows merely from the fact that $1+H^{2} \geq 1$. Equality is achieved in the final inequality of (11) for every member of the family $\mathbb{T}_{r}$ of tori defined in (10). In contrast, only the Clifford torus amongst these tori manages equality throughout (10). The entire class of flat tori in $S^{3}$ extends well beyond the family $\mathbb{T}_{r}$ (and its images under isometries of $S^{3}$ ). Pinkall [19] has observed that the preimage of a closed curve in $S^{2}$ under the Hopf fibration $S^{3} \rightarrow S^{2}$ is a flat torus - and many of these tori turn out to be Willmore surfaces. From a different perspective, any Lagrangian torus in $\mathbb{R}^{4}$ taking values in $S^{3}$ must be flat (see Remark 2 for a proof using the Gauss map, or simply use the Lagrangian structure to map the exterior normal vector of $S^{3}$ in $\mathbb{R}^{4}$ to a parallel vector field on the immersed surface). In particular, the Willmore conjecture holds for any Lagrangian surface in $\mathbb{R}^{4}$ taking values in $S^{3}$. In fact, the Willmore conjecture holds for a fairly large class of Lagrangian tori satisfying certain Maslov class restrictions (see Remark 2).

Our next result, also a corollary of a later estimate, demonstrates a certain robustness in the requirement of vanishing Gauss curvature, requiring instead an upper bound for the total curvature.

Theorem 2 Any immersed torus in $S^{3}$ satisfying

$$
\int_{\mathcal{M}}|K| \leq \frac{2}{\pi} \int_{\mathcal{M}} H^{2}
$$

obeys the Willmore conjecture

$$
W=\int_{\mathcal{M}}\left(1+H^{2}\right) \geq 2 \pi^{2} .
$$

It is worth pointing out that once the hypothesis (12) is satisfied, Theorem 2 is only useful when the total curvature (the left-hand side of (12)) is less than $4 \pi$, since otherwise we may estimate

$$
W=\int_{\mathcal{M}}\left(1+H^{2}\right) \geq \int_{\mathcal{M}} H^{2} \geq \frac{\pi}{2} \int_{\mathcal{M}}|K| \geq 2 \pi^{2} .
$$

In contrast, if the total curvature is at least $4 \pi^{2}$, then we do not even require (12) since then (8) implies

$$
W=\int_{\mathcal{M}}\left(1+H^{2}\right) \geq \int_{\mathcal{M}_{+}} K=\frac{1}{2} \int_{\mathcal{M}}|K| \geq 2 \pi^{2} .
$$

The source of these corollaries is a sharp curvature estimate which applies to surfaces of any genus, but which lies at a slight angle to the Willmore conjecture, since it pays less respect to the conformal invariance of $W$. 
Theorem 3 Given an immersed surface in $S^{3}$ of genus $g$, there holds the estimate

$$
\pi^{2}(1+g) \leq \int_{\mathcal{M}}\left(\frac{K}{2} \sin ^{-1}\left(\frac{K}{2 \sqrt{H^{2}+1-K+\frac{K^{2}}{4}}}\right)+\sqrt{1+H^{2}-K}\right),
$$

where $\sin ^{-1}$ takes a value in $\left(-\frac{\pi}{2}, \frac{\pi}{2}\right)$, which implies

$$
\pi^{2}(1+g) \leq \int_{\mathcal{M}}\left(\frac{\pi}{4}|K|+\sqrt{1+H^{2}-K}\right) .
$$

Theorems 1 and 2 follow easily from Theorem 3. For the latter, we observe that the integrand of (15) obeys

$$
\begin{aligned}
\sqrt{1+H^{2}-K}+\frac{\pi}{4}|K| & \leq \frac{1}{2}+\frac{1}{2}\left(1+H^{2}-K\right)+\frac{\pi}{4}|K| \\
& =1+H^{2}-\frac{K}{2}+\left(\frac{\pi}{4}|K|-\frac{1}{2} H^{2}\right),
\end{aligned}
$$

and so for $g=1$, (15) implies

$$
2 \pi^{2} \leq \int_{\mathcal{M}}\left(\left(1+H^{2}\right)+\frac{\pi}{4}\left(|K|-\frac{2}{\pi} H^{2}\right)\right)
$$

which is a little stronger than Theorem 2.

It may be checked directly, although it will become clear later for geometric reasons, that if the polar map $u^{*}$ is also an immersion (in other words if the principal curvatures are nowhere vanishing) then the quantity on the right-hand side of (14) for $u$ is identical to the same quantity for $u^{*}$.

Depending on the proof chosen for Theorem 3, the left-hand side of (14) arises as either the volume, or renormalised volume, of $S^{3}$ (whose volume is $2 \pi^{2}$ ) or of $S O(3)$. The proof we emphasise in this article ends up resembling work of Chern and Lashof (see [8]); their ideas have been used already in this context by Chen [7]. A similar approach has been developed independently by Cai [5] with different conclusions drawn. The methods we use in the proof will help set the scene for the proofs of some of our later results.

We remark that equality is achieved in (14) both for any round 2-sphere (of any size) in $S^{3}$, and for all of the family $\mathbb{T}_{r}$ of tori. Note that the second term on the right-hand side of (14) is zero in the case of round spheres, whereas the first term is zero for the tori $\mathbb{T}_{r}$. In fact, equality is achieved precisely for 'tight' surfaces in $S^{3} \hookrightarrow \mathbb{R}^{4}$ (which correspond to 'taut' surfaces in $\mathbb{R}^{3}$ ) and according to Banchoff [2] the only tight surfaces in $S^{3}$ are round 2-spheres and the images of the tori $\mathbb{T}_{r}$ under conformal transformations of the ambient $S^{3}$. Tight surfaces in $S^{3} \hookrightarrow \mathbb{R}^{4}$ may be defined to be the surfaces on which the restriction of any linear function on the ambient 
$\mathbb{R}^{4}$ has (if it is a Morse function) the minimum number $2(g+1)$ of critical points allowed by Morse theory. Analogously, a taut surface in $\mathbb{R}^{3}$ is one on which the restriction of any distance function from a fixed point in $\mathbb{R}^{3}$ has (if it is a Morse function) the minimum number $2(g+1)$ of critical points. Further details may be found in the book of Cecil and Ryan [6].

Rephrased slightly, tight surfaces in $S^{3} \hookrightarrow \mathbb{R}^{4}$ have the minimum number of tangency points to any foliation of parallel planes in the ambient $\mathbb{R}^{4}$, whilst taut surfaces in $\mathbb{R}^{3}$ have the minimum number of tangency points to any foliation of concentric 2 -spheres. For our second series of results, we extend these notions to consider the number of points of tangency not to planes or spheres, but to the family of tori $\mathbb{T}_{r}$ and its rotations.

The Grassmannian $G_{2,4}$ of oriented 2-planes in $\mathbb{R}^{4}$ is equivalent, via intersection with $S^{3}$, to the space of oriented great circles in $S^{3}$. Up to a normalisation, which need not concern us for the moment, there is a unique metric on $G_{2,4}$ which is invariant under the natural action of $O(4)$ in the sense that for any $M \in O(4)$, the induced map $M: G_{2,4} \rightarrow G_{2,4}$ is an isometry. This metric is constructed explicitly later - see Remark 1 .

To each oriented great circle in $G_{2,4}$, we associate a foliation of tori $\mathbb{T}_{r}$ with the great circle arising as $\mathbb{T}_{0}$. We may then define the tangency map

$$
\Phi: G_{2,4} \rightarrow \mathbb{N} \cup\{0, \infty\}
$$

of some immersed surface in $S^{3}$, to map each oriented great circle to the number of points of tangency between its associated foliation of tori $\mathbb{T}_{r}$ and the surface itself. We include in this count any points where the surface is tangent to either $\mathbb{T}_{0}$ or $\mathbb{T}_{1}$. Clearly $\Phi$ is invariant under both changes of orientation of the great circle to which it applies, and transformation of the circle to one of the two orthogonal great circles, since all four circles induce the same foliation of tori. (By orthogonal great circles, we mean here two great circles which lie in 2-planes in $\mathbb{R}^{4}$ which are the orthogonal complement of each other.)

In the case that the immersed surface is a round 2-sphere in $S^{3}$, we assert that $\Phi$ is equal to 2 almost everywhere (see Sect. 4.3) and in general for a genus $g$ surface, $\Phi$ will be at least $2(g+1)$ almost everywhere (via a Morse theory argument which will be made precise in Sect. 4.3). However, for the Clifford torus in $S^{3}$, it will become clear that $\Phi$ is equal to 8 almost everywhere - twice its minimum value given by Morse theory (this too will be discussed in Sect. 4.3). It is worth mentioning that whilst the notions of tightness and tautness are invariant under conformal transformations of the ambient space (foliations of spheres are mapped to foliations of spheres under conformal transformations) the map $\Phi$ is capable of detecting such transformations; indeed, as the Clifford torus is conformally mapped (by a homothety) into a very small region of $S^{3}$, the value of $\Phi$ will, in the 
limit, drop at almost every point in $G_{2,4}$ to 4 . (Note that the foliation of tori $\mathbb{T}_{r}$ looks like a foliation of planes to a miniature surface in $S^{3}$, and the conformally shrunk Clifford torus is tight - and therefore has 4 points of tangency with such foliations.)

It turns out that we can control the Willmore energy in terms of the average value of $\Phi$ with respect to the invariant metric on $G_{2,4}$.

Theorem 4 Given an immersed torus in $S^{3}$, its Willmore energy is bound by

$$
W \geq \frac{\pi^{2}}{4}\left(f_{G_{2,4}} \Phi\right) .
$$

In particular, the Willmore conjecture holds for any torus for which the average value of $\Phi$ is at least 8 . We have already remarked that this is the case for the Clifford torus, but that in general we only know that $\Phi$ is pointwise at least 4 . However, there is hope that any torus, once transformed to lie evenly throughout $S^{3}$ in some sense, will have an average value of $\Phi$ of at least 8 , which would solve the Willmore conjecture completely.

One situation in which we can verify that the average value of $\Phi$ is at least 8 is when the torus is invariant under the antipodal map $\mathbf{x} \rightarrow-\mathbf{x}$ of the ambient $S^{3}$. We then recover a recent result of Ros [20]:

Corollary 5 Any immersed torus in $S^{3}$ which remains invariant when composed with the antipodal map of $S^{3}$ obeys the Willmore conjecture

$$
W=\int_{\mathcal{M}}\left(1+H^{2}\right) \geq 2 \pi^{2} .
$$

In fact, with this antipodal invariance, we find the pointwise bound $\Phi \geq 8$ as we describe in Sect. 4.3. Although we suppress the details, the bound for $\Phi(p)$ may be improved, giving an improvement in Corollary 5, in terms of the number of times (in excess of four) that the plane $p$ cuts the torus $\mathcal{M}$.

The proof of Theorem 4 involves integral geometric constructions related to the Gauss map $\mathcal{G}$. The value $2 \pi^{2}$ arises as a renormalised volume of the entire Grassmannian $G_{2,4} \cong S^{2} \times S^{2}$ (see Sect. 4.2).

One significant difference between Theorems 3 and 4 , as we shall see, is that Theorem 3 has the most to say about the Willmore conjecture when the total curvature of the surface (the integral of $|K|$ ) is small, whereas Theorem 4 could in theory be strengthened for surfaces with nonzero total curvature. Our third result resembles Theorem 3 in this respect; the estimate we achieve has an error term involving the least energy of a unit tangent vector field (with a certain topology) on the surface, and this term will be zero for flat tori.

The principle behind this result was alluded to in the motivation discussed in Sect. 1. We expect any reasonable notion of Gauss map to be related to 
the Willmore energy via its harmonic energy (cf. the calculations of Sect. 1). Therefore, if a Gauss map of a surface lies in a nontrivial homotopy class then we expect to be able to bound the Willmore energy of the surface from below in terms of the infimum of the harmonic energy within the homotopy class of maps represented by the Gauss map. Unfortunately, the notions $u^{*}$ and $\mathcal{G}$ of Gauss map we have up to now give homotopically trivial maps when the surface is a torus. To combat this handicap, we use the fact that tori admit nowhere vanishing tangent vector fields, and extend the notion of Gauss map to incorporate this, giving finally a map into $S O(4)$ which is not trivial. The price for this topological trick is paid by the geometry - the harmonic energy of the Gauss map incorporates a term representing the energy of the vector field, and this persists as an error term in the final estimate.

We define the energy of a unit (tangent) vector field $v: \mathcal{M} \rightarrow S^{3} \hookrightarrow \mathbb{R}^{4}$, for an immersion $u: \mathcal{M} \rightarrow S^{3} \hookrightarrow \mathbb{R}^{4}$, to be

$$
\mathcal{E}(v)=\frac{1}{2} \int_{\mathcal{M}}\left(\left\langle\frac{\partial v}{\partial x}, w\right\rangle^{2}+\left\langle\frac{\partial v}{\partial y}, w\right\rangle^{2}\right) d x \wedge d y,
$$

where $x$ and $y$ are isothermal coordinates on $\mathcal{M}$ and $w: \mathcal{M} \rightarrow S^{3} \hookrightarrow \mathbb{R}^{4}$ is a unit tangent vector field which is everywhere orthogonal to $v$. The energy measures the twisting of the vector field $v$.

In the following result, we require some compatibility between the topology of the vector fields considered, the topology of the torus immersion, and the conformal class of the torus. We have not sought the most general statement possible, choosing instead to consider a simple case where this compatibility is easily verified.

Theorem 6 Let $\Gamma$ be the lattice in $\mathbb{R}^{2}$ generated by $(1,0)$ and $(0, d)$, for some $d>0$, and let $\mathcal{M}$ be the torus $\mathbb{R}^{2} / \Gamma$. Suppose that $u: \mathcal{M} \rightarrow$ $S^{3}$ is a conformal immersion which may be deformed through a family of immersions $\mathcal{M} \rightarrow S^{3}$ to the Clifford torus immersion

$$
u_{0}(x, y)=\frac{1}{\sqrt{2}}\left(\cos 2 \pi x, \sin 2 \pi x, \cos \frac{2 \pi y}{d}, \sin \frac{2 \pi y}{d}\right) .
$$

We denote by $\Sigma$ the homotopy class of unit vector fields on the immersed torus which contains the representative $\frac{\partial u}{\partial x} /\left|\frac{\partial u}{\partial x}\right| ;$ more explicitly, we have

$$
\Sigma:=\left\{\frac{1}{\left|\frac{\partial u}{\partial x}\right|}\left(\cos \theta \frac{\partial u}{\partial x}+\sin \theta \frac{\partial u}{\partial y}\right) \mid \theta: \mathcal{M} \rightarrow \mathbb{R} \text { is regular }\right\} .
$$

Then for any $v \in \Sigma$, the Willmore energy satisfies

$$
W+\frac{1}{2} \mathcal{E}(v) \geq \pi^{2}\left(d+\frac{1}{d}\right) \geq 2 \pi^{2} .
$$


When the immersed torus given by $u$ has zero Gauss curvature, the quantity $\left|\frac{\partial u}{\partial x}\right|=\left|\frac{\partial u}{\partial y}\right|$ is constant and we may set $v=\frac{\partial u}{\partial x} /\left|\frac{\partial u}{\partial x}\right|$ to force $\mathcal{E}(v)=0$ and establish the goal $W \geq 2 \pi^{2}$. If $\mathcal{M}$ is one of the flat tori $\mathbb{T}_{r}$, and we choose $v$ in this manner (so that $\mathcal{E}(v)=0$ ) then we have equality in the first inequality of (17), with equality throughout (17) for the Clifford torus $\mathbb{T}_{\frac{1}{\sqrt{2}}}$.

As a final remark, we mention that some of the observations in this work allow progress towards the Willmore conjecture for Lagrangian surfaces in $\mathbb{R}^{4}$. Elaboration may be found in Remark 2 towards the end of the paper.

\section{Proofs of the results}

\subsection{Volume sweeping in $S^{3}$ - the proof of Theorem 3}

Let us begin with a few preliminary definitions which will serve us beyond the proof of Theorem 3. Although we will only later be concerned with the orientations on the objects we define, we set

$$
\mathcal{S}=\left\{\text { oriented great circles in } S^{3}\right\} .
$$

The space $\mathcal{S}$ may be identified with the space $G_{2,4}$ of oriented 2-planes in $\mathbb{R}^{4}$ by intersecting each plane with $S^{3}$ and allowing the orientation to be induced. We also will require the the identification of $\mathcal{S}=G_{2,4}$ with

$$
\mathcal{P}=\left\{e \wedge f \mid e, f \text { are orthogonal unit vectors in } \mathbb{R}^{4}\right\} \subset \wedge^{2}\left(\mathbb{R}^{4}\right),
$$

where an element $e \wedge f \in \mathcal{P}$ corresponds to the plane $\operatorname{span}(e, f)$, with $\{e, f\}$ being a positively oriented basis, in $G_{2,4}$. These identifications will be used without referring explicitly to the identification maps; for example we are liable to talk of a plane or circle $e \wedge f$, and may apply the Hodge *-operator to a plane (giving the orthogonal-complement plane).

Using the identifications above, we may define a map $\Lambda: \mathcal{M} \rightarrow S$ corresponding to the bipolar map

$$
x \rightarrow u(x) \wedge u^{*}(x)
$$

from $\mathcal{M}$ to $\mathcal{P}$. Each point $x \in \mathcal{M}$ is therefore sent to one of the two oriented great circles passing through $u(x)$ and $u^{*}(x)$.

As we move the point $x$ on the two-dimensional surface, the one-dimensional great circle it is mapped to under $\Lambda$ sweeps out a three-dimensional volume in $S^{3}$. The strategy of the proof is to obtain an expression for the volume being swept in terms of the local curvature properties of $\mathcal{M}$, and then use the topology of $\mathcal{M}$ to prove that the entire $S^{3}$ is swept over at least $2(1+g)$ times, where $g$ is the genus of $\mathcal{M}$. 
The amount of volume swept out as $x$ moves within a Borel set $A \subset \mathcal{M}$ (counted with geometric multiplicity) is given by a measure

$$
\mu(A)=\int_{S^{3}} \mathcal{H}^{0}\{x \in A \mid y \text { lies on } \Lambda(x)\} d y,
$$

where $\mathcal{H}^{0}$ represents the 0 -Hausdorff measure, and counts the number of points in the set it is measuring. The measure $\mu$ may then be written as

$$
\mu(A)=\int_{A} h
$$

where $h: \mathcal{M} \rightarrow \mathbb{R}$ is a non-negative function for which we now seek an explicit expression in terms of the Gauss and mean curvatures. Let us choose 'adapted' isothermal coordinates $x$ and $y$ near some point $p \in \mathcal{M}$ (with $(x, y)=(0,0)$ corresponding to the point $p)$ and orthonormal coordinates (with positive orientation) for $\mathbb{R}^{4} \hookleftarrow S^{3}$, so that

$$
u(0,0)=(1,0,0,0), \quad \text { and } \quad u^{*}(0,0)=(0,1,0,0),
$$

and so that

$$
\frac{\partial u}{\partial x}(0,0)=(0,0,1,0) \quad \text { and } \quad \frac{\partial u}{\partial y}(0,0)=(0,0,0,1)
$$

are required to represent the principal curvature directions corresponding to $\kappa_{1}$ and $\kappa_{2}$ respectively (provided $p$ is not an umbilic point). Observe then that

$$
\frac{\partial u^{*}}{\partial x}(0,0)=\left(0,0, \kappa_{1}, 0\right) \quad \text { and } \quad \frac{\partial u^{*}}{\partial y}(0,0)=\left(0,0,0, \kappa_{2}\right) .
$$

The image of $p$ under $\Lambda$ is then the great circle

$$
\{(\cos \theta, \sin \theta, 0,0) \mid \theta \in[0,2 \pi)\} .
$$

More generally, we define $\bar{\Lambda}: \mathcal{M} \times[0,2 \pi) \rightarrow S^{3} \hookrightarrow \mathbb{R}^{4}$ by

$$
\bar{\Lambda}(x, y, \theta)=\cos \theta u(x, y)+\sin \theta u^{*}(x, y),
$$

so that the image of $\bar{\Lambda}(x, y, \cdot)$ gives the great circle $\Lambda(x, y)$. Then we clearly have

$$
\frac{\partial \bar{\Lambda}}{\partial x}(0,0, \theta)=\cos \theta \frac{\partial u}{\partial x}(0,0)+\sin \theta \frac{\partial u^{*}}{\partial x}(0,0)=\left(0,0, \cos \theta+\kappa_{1} \sin \theta, 0\right),
$$

and

$$
\frac{\partial \bar{\Lambda}}{\partial y}(0,0, \theta)=\cos \theta \frac{\partial u}{\partial y}(0,0)+\sin \theta \frac{\partial u^{*}}{\partial y}(0,0)=\left(0,0,0, \cos \theta+\kappa_{2} \sin \theta\right),
$$


and consequently

$$
h(0,0)=\int_{0}^{2 \pi}\left|\left(\cos \theta+\kappa_{1} \sin \theta\right)\left(\cos \theta+\kappa_{2} \sin \theta\right)\right| d \theta .
$$

An elementary calculation, using the relationship between $\kappa_{1}, \kappa_{2}, H$ and $K$ reveals that

$$
\begin{gathered}
\left(\cos \theta+\kappa_{1} \sin \theta\right)\left(\cos \theta+\kappa_{2} \sin \theta\right)=\frac{K}{2}+\left(1-\frac{K}{2}\right) \cos 2 \theta+H \sin 2 \theta \\
=\frac{K}{2}+\sqrt{H^{2}+\left(1-\frac{K}{2}\right)^{2}} \cos (2 \theta+\alpha),
\end{gathered}
$$

for some $\alpha=\alpha(H, K)$. Observing, with the aid of (8), that

$$
\left|\frac{K}{2}\right| \leq \sqrt{H^{2}+1-K+\frac{K^{2}}{4}}=\sqrt{H^{2}+\left(1-\frac{K}{2}\right)^{2}},
$$

(and thus that the right-hand side of (22) changes sign) we may then use (22) to evaluate the integral in (21), eventually yielding

$$
h=4\left[\frac{K}{2} \sin ^{-1}\left(\frac{K}{2 \sqrt{H^{2}+1-K+\frac{K^{2}}{4}}}\right)+\sqrt{H^{2}+1-K},\right.
$$

where $\sin ^{-1}$ takes values in $\left(-\frac{\pi}{2}, \frac{\pi}{2}\right)$.

Now we turn our attention to a lower bound for $\mu(\mathcal{M})$. We claim that the great circles given by $\Lambda$ sweep over the whole of $S^{3}$ at least $2(1+g)$ times, and hence that

$$
\mu(\mathcal{M}) \geq 2(1+g) \operatorname{vol}\left(S^{3}\right)=4 \pi^{2}(1+g) .
$$

It suffices to prove that for almost all $\xi \in S^{3}$ there are at least $2(1+g)$ points $x_{i} \in \mathcal{M}$ such that $\xi$ lies on each circle $\Lambda\left(x_{i}\right)$. Indeed, we will show that this is the case for any $\xi \in S^{3} \hookrightarrow \mathbb{R}^{4}$ for which the function $f: \mathcal{M} \rightarrow \mathbb{R}$ defined using the inner product on $\mathbb{R}^{4}$ by

$$
f(p)=\langle u(p), \xi\rangle
$$

is a Morse function; only the focal points of $\mathcal{M}$ (a negligible codimension one set in $S^{3}$ ) fail this criterion. Now if $f$ is a Morse function, Morse theory tells us (see [16] for example) that $f$ must have at least $2(1+g)$ critical points $(2(1+g)$ being the sum of the Betti numbers of $\mathcal{M})$. Since the critical points of $f$ are precisely the points at which the tangent space of the surface lies perpendicular to the vector $\xi$, at each critical point $p \in \mathcal{M}$ the circle $\Lambda(p)$ 
perpendicular to the surface must contain $\xi$ as desired. To summarise, for almost all $\xi \in S^{3}$ we have

$$
\mathcal{H}^{0}\{x \in \mathcal{M} \mid \xi \text { lies on } \Lambda(x)\} \geq 2(1+g),
$$

and hence we have established (24). Combining the information on $\mu(\mathcal{M})$ given by (24) and (23), we arrive at (14) and complete the proof of Theorem 3.

Towards the end of Sect. 4, a second approach to the proof of this theorem will be sketched in which the integral geometry is performed on $S O(3)$ rather than $S^{3}$.

\subsection{Integral geometry on $G_{2,4}$ - the proof of Theorem 4}

Progress in this section is based on understanding the structure of the Grassmannian $G_{2,4}=\mathcal{P}$, and forcing the Gauss map, or the bipolar map, to be distributed sufficiently evenly within.

We begin by giving a natural identification between $\mathcal{P}$ and the product $S^{2} \times S^{2}$. Let us set the inner product on $\bigwedge^{2}\left(\mathbb{R}^{4}\right)$, in which $\mathcal{P}$ lies, to be

$$
\left\langle\sum_{i<j} c_{i j} e_{i} \wedge e_{j}, \sum_{i<j} d_{i j} e_{i} \wedge e_{j}\right\rangle=\sum_{i<j} c_{i j} d_{i j},
$$

where $\left\{e_{1}, \ldots, e_{4}\right\}$ is an orthonormal basis for $\mathbb{R}^{4}$. Given an element $e \wedge f$ of $\mathcal{P}$ with $e$ and $f$ orthogonal unit vectors in $\mathbb{R}^{4}$, we may then take the projections $p_{+}: \mathcal{P} \rightarrow \bigwedge_{+}^{2}\left(\mathbb{R}^{4}\right)$ and $p_{-}: \mathcal{P} \rightarrow \bigwedge_{-}^{2}\left(\mathbb{R}^{4}\right)$ onto the eigenspaces

$$
\bigwedge_{+}^{2}\left(\mathbb{R}^{4}\right)=\left\{\omega \in \bigwedge^{2}\left(\mathbb{R}^{4}\right) \mid * \omega=\omega\right\}
$$

and

$$
\bigwedge_{-}^{2}\left(\mathbb{R}^{4}\right)=\left\{\omega \in \bigwedge^{2}\left(\mathbb{R}^{4}\right) \mid * \omega=-\omega\right\}
$$

of the Hodge star operator $*: \bigwedge^{2}\left(\mathbb{R}^{4}\right) \rightarrow \bigwedge^{2}\left(\mathbb{R}^{4}\right)$. Explicitly, $p_{+}(e \wedge f)=$ $\frac{1}{2}(e \wedge f+*(e \wedge f))$ and $p_{-}(e \wedge f)=\frac{1}{2}(e \wedge f-*(e \wedge f))$. In this way, we see that although $\mathcal{P}$ clearly lies on the 5 -sphere within the six dimensional $\bigwedge^{2}\left(\mathbb{R}^{4}\right)$, it is further constrained to lie on a product

$$
\left(\frac{1}{\sqrt{2}} S^{2}\right) \times\left(\frac{1}{\sqrt{2}} S^{2}\right) \hookrightarrow S^{5} .
$$

Indeed, since the projections $p_{+}$and $p_{-}$map $\mathcal{P}$ into the 2 -spheres in $\bigwedge_{+}^{2}\left(\mathbb{R}^{4}\right)$ and $\bigwedge_{-}^{2}\left(\mathbb{R}^{4}\right)$ centred at the origin and with radius $\frac{1}{\sqrt{2}}$, we may define a map

$$
\alpha: G_{2,4}=\mathcal{P} \rightarrow S^{2} \times S^{2},
$$

defined by

$$
\alpha=\left(\sqrt{2} p_{+}, \sqrt{2} p_{-}\right)
$$


which is then a bijection.

Note that given an element $p \in G_{2,4}=\mathcal{P}$, if

$$
\alpha(p)=(a, b) \in S^{2} \times S^{2} \hookrightarrow \mathbb{R}^{3} \times \mathbb{R}^{3},
$$

then $\alpha(-p)=(-a,-b)$ and and $\alpha(* p)=(a,-b)$.

Remark 1 If we pull back the standard metric on $S^{2} \times S^{2}$ to $G_{2,4}$ using $\alpha$, we get the metric to be used in the integral of Theorem 4

We adopt the following orientations on $\bigwedge_{+}^{2}\left(\mathbb{R}^{4}\right)$ and $\bigwedge_{-}^{2}\left(\mathbb{R}^{4}\right)$, and hence on the target of $\alpha$. Given a positively oriented orthonormal basis $\left\{e_{1}, \ldots, e_{4}\right\}$ of $\mathbb{R}^{4}$, we declare the orthonormal bases

$$
\begin{gathered}
\left\{\frac{1}{\sqrt{2}}\left(e_{2} \wedge e_{3}+e_{1} \wedge e_{4}\right), \frac{1}{\sqrt{2}}\left(e_{3} \wedge e_{1}+e_{2} \wedge e_{4}\right),\right. \\
\left.\quad \frac{1}{\sqrt{2}}\left(e_{1} \wedge e_{2}+e_{3} \wedge e_{4}\right)\right\}
\end{gathered}
$$

of $\bigwedge_{+}^{2}\left(\mathbb{R}^{4}\right)$, and

$$
\begin{gathered}
\left\{\frac{1}{\sqrt{2}}\left(e_{2} \wedge e_{3}-e_{1} \wedge e_{4}\right), \frac{1}{\sqrt{2}}\left(e_{3} \wedge e_{1}-e_{2} \wedge e_{4}\right),\right. \\
\left.\quad \frac{1}{\sqrt{2}}\left(e_{1} \wedge e_{2}-e_{3} \wedge e_{4}\right)\right\}
\end{gathered}
$$

of $\bigwedge_{-}^{2}\left(\mathbb{R}^{4}\right)$ to be positively oriented. That this declaration is well defined will shortly become clear.

Elements of $S O(4)$ act naturally on the space $G_{2,4}$. It will be important to understand the exact effect this action has on the target $S^{2} \times S^{2}$ of $\alpha$. In other words, given an element $M \in S O(4)$, we would like to understand the map $S^{2} \times S^{2}$ to $S^{2} \times S^{2}$ given by $\alpha \circ M \circ \alpha^{-1}$. It is not hard to see that the action of $S O(4)$ on $\mathcal{P}=G_{2,4}$ is given by an orthogonal transformation acting on the six dimensional $\bigwedge^{2}\left(\mathbb{R}^{4}\right)$. This transformation then reduces to an element of $S O(3) \times S O(3)$ acting on $\bigwedge_{+}^{2}\left(\mathbb{R}^{4}\right) \oplus \bigwedge_{-}^{2}\left(\mathbb{R}^{4}\right)$ which we now describe explicitly.

It is well known (see [9]) that any element $M \in S O(4)$ may be decomposed into a simultaneous rotation in some plane in $\mathbb{R}^{4}$ and in its orthogonalcomplement plane. We therefore choose two such 2-planes $p, q \in G_{2,4}=\mathcal{P}$ such that $* p=q$ (and so $* q=p$ ) and so that the rotation in $p$ is by an angle $\theta$ and the rotation in $q$ is by an angle $\varphi$. Here we fix the signs of the angles $\theta$ and $\varphi$ so that if $p=e \wedge f$ with $e$ and $f$ orthogonal unit vectors in $\mathbb{R}^{4}$, then an infinitesimal positive rotation in the plane moves $e$ in the direction of $f$.

Now it is clear that the element of $(A, B) \in S O(3) \times S O(3)$ to which $\alpha \circ M \circ \alpha^{-1}$ corresponds, must fix the point $\alpha(p) \in S^{2} \times S^{2}$. Writing 
$\alpha(p)=(a, b) \in S^{2} \times S^{2}$, we have $A$ a rotation about the axis through the origin and $a$, and $B$ a rotation about the axis through the origin and $b$. It remains to establish the angles of these rotations. By direct calculation, or consideration of infinitesimal rotations, we see that $A$ is a rotation by $\theta+\varphi$, whilst $B$ is a rotation by $\theta-\varphi$. Here, the sign of a rotation about an axis through $a$ is fixed to make a positive infinitesimal rotation move a vector $e$ in the direction of $f$ whenever $\{e, f, a\}$ form an orthonormal basis for $\mathbb{R}^{3} \hookleftarrow S^{2}$.

We will write the map sending $M \in S O(4)$ to $(A, B) \in S O(3) \times S O(3)$ as

$$
\gamma: S O(4) \rightarrow S O(3) \times S O(3) .
$$

The kernel of $\gamma$ as a group homomorphism consists of the identity $I$ and the antipodal map $-I$. After endowing the compact Lie groups $S O(3)$ and $S O(4)$ with appropriate invariant metrics, the map $\gamma$ is a locally isometric two-sheeted covering. By 'invariant' metric we mean one for which both the left and right group actions are isometries. There is a unique such metric, up to a normalisation constant, for $S O(3)$.

Note that orientation reversing elements of $O(4)$ have the additional effect of interchanging the 2 -spheres in the target $S^{2} \times S^{2}$ of $\alpha$. For example, a reflection about the hyperplane orthogonal to $e_{4}$ will, using the bases in (26) and (27), map each plane $\alpha^{-1}(a, b)$ to the plane $\alpha^{-1}(b, a)$.

Using the identification given by $\alpha$, we see the Gauss map as a map

$$
\sigma=\alpha \circ \mathcal{G}: \mathcal{M} \rightarrow S^{2} \times S^{2} .
$$

We decompose this as $\sigma=(a, b)$ where $a, b: \mathcal{M} \rightarrow S^{2}$ are the compositions of $\sigma$ with the projections onto the two copies of $S^{2}$. Our choice of orientation for the 2 -spheres will then make the Jacobian of both $a$ and $b$ everywhere equal to the Gauss curvature $K$ (as we shall shortly justify) at which point the Gauss-Bonnet formula forces

$$
\operatorname{deg}(a)=\operatorname{deg}(b)=1-g .
$$

For example, if the original surface is a round 2-sphere, then both $a$ and $b$ are the identity map (up to rotation and expansion). We will also have that the harmonic energy density of both $a$ and $b$ is given by

$$
\frac{1}{2}|\nabla a|^{2}=\frac{1}{2}|\nabla b|^{2}=2+2 H^{2}-K,
$$

and therefore for tori, we deduce that

$$
E(a)=E(b)=2 W .
$$

The harmonic energy of $\sigma$ for tori is then clearly

$$
E(\sigma)=E(a)+E(b)=4 W,
$$


and bounds the geometric area

$$
\operatorname{Area}(\operatorname{Image}(\sigma))=\int_{\mathcal{M}} 2\left[\left(1+\kappa_{1}^{2}\right)\left(1+\kappa_{2}^{2}\right)\right]^{\frac{1}{2}},
$$

of the image of $\sigma$ from above.

To verify these connections between the curvature of the surface and the area and harmonic energy of the maps $a$ and $b$, we calculate explicitly. Given a point $p \in \mathcal{M}$, let us choose an orthonormal basis $\left\{e_{1}, \ldots, e_{4}\right\}$ for $\mathbb{R}^{4}$ and adapted isothermal coordinates $x$ and $y$ near $p$, in order to satisfy (18), (19) and (20) as in Sect. 4.1. Therefore, we have

$$
\begin{gathered}
\left(u \wedge u^{*}\right)(0,0)=e_{1} \wedge e_{2}, \frac{\partial\left(u \wedge u^{*}\right)}{\partial x}(0,0)=e_{3} \wedge e_{2}+\kappa_{1} e_{1} \wedge e_{3}, \\
\frac{\partial\left(u \wedge u^{*}\right)}{\partial y}(0,0)=e_{4} \wedge e_{2}+\kappa_{2} e_{1} \wedge e_{4},
\end{gathered}
$$

and since the Gauss map $\mathcal{G}$ is given by $*\left(u \wedge u^{*}\right)$, this yields

$$
\begin{gathered}
\mathcal{G}(0,0)=e_{3} \wedge e_{4}, \frac{\partial \mathcal{G}}{\partial x}(0,0)=e_{4} \wedge e_{1}+\kappa_{1} e_{4} \wedge e_{2}, \\
\frac{\partial \mathcal{G}}{\partial y}(0,0)=e_{1} \wedge e_{3}+\kappa_{2} e_{2} \wedge e_{3},
\end{gathered}
$$

where we are using implicitly, as usual, the equivalence of $\mathcal{P}$ and $G_{2,4}$. Projecting onto $\bigwedge_{+}^{2}\left(\mathbb{R}^{4}\right)$ and $\bigwedge_{-}^{2}\left(\mathbb{R}^{4}\right)$ and using coordinates with respect to the bases given in (26) and (27) (with appropriate scaling) we have $\sigma$ given by

$$
\begin{gathered}
a(0,0)=(0,0,1), \quad \frac{\partial a}{\partial x}(0,0)=\left(-1,-\kappa_{1}, 0\right), \\
\frac{\partial a}{\partial y}(0,0)=\left(\kappa_{2},-1,0\right),
\end{gathered}
$$

and

$$
\begin{gathered}
b(0,0)=(0,0,-1), \quad \frac{\partial b}{\partial x}(0,0)=\left(1, \kappa_{1}, 0\right), \\
\frac{\partial b}{\partial y}(0,0)=\left(\kappa_{2},-1,0\right) .
\end{gathered}
$$

At this point we may evaluate the Jacobians of both $a$ and $b$ to be $1+\kappa_{1} \kappa_{2}=$ $K$, whilst the harmonic energy densities are given by

$$
e(a):=\frac{1}{2}|\nabla a|^{2}=\frac{1}{2}\left(\left(1+\kappa_{1}^{2}\right)+\left(1+\kappa_{2}^{2}\right)\right)=2+2 H^{2}-K,
$$

and likewise for $b$. 
It may be worth illustrating these calculations with examples. If the surface $\mathcal{M}$ is the Clifford torus in $S^{3}$ given by the isometry

$$
(x, y) \rightarrow \frac{1}{\sqrt{2}}(\cos \sqrt{2} x, \sin \sqrt{2} x, \cos \sqrt{2} y, \sin \sqrt{2} y),
$$

with $x$ and $y$ ranging over the interval $(0, \sqrt{2} \pi)$, then by direct calculation, we find that (taking $\left\{\frac{\partial u}{\partial x}, \frac{\partial u}{\partial y}\right\}$ to be a positive basis for the tangent space) the Gauss map is given by

$$
\begin{aligned}
\mathcal{G}(x, y)= & {\left[-\sin (\sqrt{2} x) e_{1}+\cos (\sqrt{2} x) e_{2}\right] \wedge\left[-\sin (\sqrt{2} y) e_{3}+\cos (\sqrt{2} y) e_{4}\right] } \\
= & \sin (\sqrt{2} x) \sin (\sqrt{2} y) e_{1} \wedge e_{3}+\cos (\sqrt{2} x) \cos (\sqrt{2} y) e_{2} \wedge e_{4} \\
& -\cos (\sqrt{2} x) \sin (\sqrt{2} y) e_{2} \wedge e_{3}-\sin (\sqrt{2} x) \cos (\sqrt{2} y) e_{1} \wedge e_{4} .
\end{aligned}
$$

As before, we now project onto $\bigwedge_{+}^{2}\left(\mathbb{R}^{4}\right)$ and $\bigwedge_{-}^{2}\left(\mathbb{R}^{4}\right)$ and use coordinates with respect to the bases given in (26) and (27) (with appropriate scaling) to find that $\sigma$ is given by

$$
a(x, y)=(-\sin \sqrt{2}(x+y), \cos \sqrt{2}(x+y), 0),
$$

and

$$
b(x, y)=(\sin \sqrt{2}(x-y),-\cos \sqrt{2}(x-y), 0) .
$$

In fact, these formulae may be deduced without calculation using our understanding of the map $\gamma: S O(4) \rightarrow S O(3) \times S O(3)$. The Clifford torus is generated by rotating the point $\frac{1}{\sqrt{2}}(1,0,1,0)$ by angles $\sqrt{2} x$ in the $e_{1} \wedge e_{2}$ plane, and $\sqrt{2} y$ in the $e_{3} \wedge e_{4}$ plane. These rotations, composed with $\gamma$, correspond to rotations by $\sqrt{2}(x+y)$ in the first $S^{2}$ (about the axis $(0,0,1)$ ) and by $\sqrt{2}(x-y)$ in the second $S^{2}$ (also about the axis $(0,0,1)$ ). Since the tangent plane at $(x, y)=(0,0)$ is $e_{2} \wedge e_{4}$, we see that $a(0,0)=(0,1,0)$ and $b(0,0)=(0,-1,0)$ and hence we deduce (31) and (32).

It is now immediate from (31) that $\frac{1}{2}|\nabla a|^{2}=2$ and hence that $E(a)=$ $4 \pi^{2}=2 W$ (and similarly for $b$ ) which verifies (28) in this case. We also observe that the Jacobians of both $a$ and $b$ are zero (they map into the equators of their target 2-spheres) and therefore agree with the zero Gauss curvature as predicted.

Now we have established the geometric properties of the map $\sigma$, we may turn our attention to performing integral geometry in the target $S^{2} \times S^{2}$. Given a point $(r, s) \in S^{2} \times S^{2}$, the associated torus of 'orthogonal' points in $S^{2} \times S^{2}$ is defined by

$$
T_{r, s}:=\left\{(k, l) \in S^{2} \times S^{2} \hookrightarrow \mathbb{R}^{3} \times \mathbb{R}^{3} \mid\langle r, k\rangle=\langle s, l\rangle=0\right\} .
$$

We denote the entire class of such tori by

$$
\mathcal{T}=\left\{T_{r, s} \mid r, s \in S^{2}\right\}
$$


In analogy to the proof of Theorem 3, we define a map $\Lambda: \mathcal{M} \rightarrow \mathcal{T}$ by

$$
\Lambda(p)=T_{a(p), b(p)},
$$

where $a$ and $b$ are the components of $\sigma$ as above. The image of $\Lambda$ is a twodimensional torus, so as we move it on the two-dimensional surface $\mathcal{M}$, it will sweep out four-dimensional volume in the four-dimensional $S^{2} \times S^{2}$. We measure this swept volume with a measure on $\mathcal{M}$ defined now by

$$
\mu(A)=\int_{S^{2} \times S^{2}} \mathcal{H}^{0}\{x \in A \mid y \text { lies on } \Lambda(x)\} d y,
$$

and as before, there exists a function $h: \mathcal{M} \rightarrow \mathbb{R}$ such that

$$
\mu(A)=\int_{A} h
$$

for any Borel set $A \subset \mathcal{M}$. The exact expression for $h$ turns out to involve elliptic integrals. However, we manage to give an explicit upper bound for it in terms of the curvatures of $\mathcal{M}$. The resulting upper bound for $\mu(\mathcal{M})$ may then be combined with an exact expression for $\mu(\mathcal{M})$ in terms of the tangency map $\Phi$ to give the desired result.

Continuing with the analogy to the proof of Theorem 3, near some point $p \in \mathcal{M}$ we pick adapted isothermal coordinates $x$ and $y$, and an orthonormal basis $\left\{e_{1}, \ldots, e_{4}\right\}$ for $\mathbb{R}^{4}$, in order to satisfy (18), (19) and (20) as in Sect. 4.1. The components $a$ and $b$ of $\sigma$, and their derivatives, are then given by (29) and (30). Meanwhile, the torus $\Lambda(p)=T_{a(p), b(p)} \hookrightarrow S^{2} \times S^{2} \hookrightarrow \mathbb{R}^{6}$ is given by

$$
\{(\cos \theta, \sin \theta, 0, \cos \varphi, \sin \varphi, 0) \mid \theta, \varphi \in[0,2 \pi)\} .
$$

More generally, we may find a parameterisation $\bar{\Lambda}: A \times[0,2 \pi) \times[0,2 \pi) \rightarrow$ $S^{2} \times S^{2} \hookrightarrow \mathbb{R}^{6}$ on a neighbourhood $A$ of $p$ such that the image of $\bar{\Lambda}(x, y, \cdot, \cdot)$ gives the torus $T_{a, b}$ at $(x, y)$, with

$$
\bar{\Lambda}(0,0, \theta, \varphi)=(\cos \theta, \sin \theta, 0, \cos \varphi, \sin \varphi, 0),
$$

and so that (keeping in mind (29) and (30))

$$
\frac{\partial \bar{\Lambda}}{\partial x}(0,0, \theta, \varphi)=\left(0,0, \cos \theta+\kappa_{1} \sin \theta, 0,0, \cos \varphi+\kappa_{1} \sin \varphi\right),
$$

and

$$
\frac{\partial \bar{\Lambda}}{\partial y}(0,0, \theta, \varphi)=\left(0,0,-\kappa_{2} \cos \theta+\sin \theta, 0,0, \kappa_{2} \cos \varphi-\sin \varphi\right) .
$$


The local expression $h$ describing the amount of swept volume is given by the integral over the torus $T_{a(p), b(p)}$ of the size of the wedge product of these derivatives:

$$
\begin{gathered}
h(0,0)=\int_{0}^{2 \pi} \int_{0}^{2 \pi} \mid\left(\cos \theta+\kappa_{1} \sin \theta\right)\left(\kappa_{2} \cos \varphi-\sin \varphi\right) \\
-\left(\cos \varphi+\kappa_{1} \sin \varphi\right)\left(-\kappa_{2} \cos \theta+\sin \theta\right) \mid d \theta d \varphi .
\end{gathered}
$$

Now since

$$
\cos \theta+\kappa_{1} \sin \theta=\sqrt{1+\kappa_{1}^{2}} \cos \left(\theta+\zeta_{1}\right)
$$

and

$$
\kappa_{2} \cos \theta-\sin \theta=\sqrt{1+\kappa_{2}^{2}} \cos \left(\theta+\zeta_{2}\right),
$$

where $\tan \zeta_{1}=-\kappa_{1}$ and $\tan \zeta_{2}=\frac{1}{\kappa_{2}}$, we have

$$
\begin{aligned}
h(0,0)= & \sqrt{\left(1+\kappa_{1}^{2}\right)\left(1+\kappa_{2}^{2}\right)} \int_{0}^{2 \pi} \int_{0}^{2 \pi} \mid \cos \left(\theta+\zeta_{1}\right) \cos \left(\varphi+\zeta_{2}\right) \\
& +\cos \left(\varphi+\zeta_{1}\right) \cos \left(\theta+\zeta_{2}\right) \mid d \theta d \varphi \\
= & \sqrt{\left(1+\kappa_{1}^{2}\right)\left(1+\kappa_{2}^{2}\right)} \int_{0}^{2 \pi} \int_{0}^{2 \pi} \mid \cos \theta \cos (\varphi+\zeta) \\
& +\cos \varphi \cos (\theta+\zeta) \mid d \theta d \varphi
\end{aligned}
$$

where $\zeta:=\zeta_{2}-\zeta_{1}$ satisfies

$$
|\tan \zeta|=\left|\frac{\tan \zeta_{2}-\tan \zeta_{1}}{1+\tan \zeta_{1} \tan \zeta_{2}}\right|=\left|\frac{1+\kappa_{1} \kappa_{2}}{\kappa_{2}-\kappa_{1}}\right|=\frac{|K|}{\sqrt{1+H^{2}-K}} .
$$

We may now calculate

$$
\begin{aligned}
f_{\zeta}(\theta, \varphi) & :=\cos \theta \cos (\varphi+\zeta)+\cos \varphi \cos (\theta+\zeta) \\
& =\cos \theta[2 \cos \varphi \cos \zeta-\sin \varphi \sin \zeta]+\sin \theta[-\cos \varphi \sin \zeta] \\
& =\cos (\theta+\beta)\left[(2 \cos \varphi \cos \zeta-\sin \varphi \sin \zeta)^{2}+\cos ^{2} \varphi \sin ^{2} \zeta\right]^{\frac{1}{2}}
\end{aligned}
$$

for some $\beta=\beta(\varphi, \zeta)$ independent of $\theta$. Expanding the part within square brackets, and rewriting the resulting expression in terms of $\cos 2 \varphi$ and $\sin 2 \varphi$, we end up with

$$
\begin{aligned}
f_{\zeta}(\theta, \varphi)= & \cos (\theta+\beta)\left[\cos 2 \varphi\left(2 \cos ^{2} \zeta\right)+\sin 2 \varphi(-2 \sin \zeta \cos \zeta)\right. \\
& \left.\quad+\cos ^{2} \zeta+1\right]^{\frac{1}{2}} \\
= & \cos (\theta+\beta)\left[\cos (2 \varphi+\delta) 2 \cos \zeta+1+\cos ^{2} \zeta\right]^{\frac{1}{2}},
\end{aligned}
$$


for some $\delta=\delta(\zeta)$. At this point, we may return to (33) to find that

$$
\begin{aligned}
h(0,0)= & \sqrt{\left(1+\kappa_{1}^{2}\right)\left(1+\kappa_{2}^{2}\right)} \int_{0}^{2 \pi} \int_{0}^{2 \pi} \mid \cos (\theta+\beta)[\cos (2 \varphi+\delta) 2 \cos \zeta \\
& \left.+1+\cos ^{2} \zeta\right]^{\frac{1}{2}} \mid d \theta d \varphi \\
= & 4 \sqrt{\left(1+\kappa_{1}^{2}\right)\left(1+\kappa_{2}^{2}\right)} \int_{0}^{2 \pi}[\cos 2 \varphi(2 \cos \zeta) \\
& \left.+1+\cos ^{2} \zeta\right]^{\frac{1}{2}} d \varphi .
\end{aligned}
$$

Although we are unable to evaluate this elliptic integral precisely, we claim that as we vary $\zeta$, the integral takes its maximum value when $\cos \zeta=1$ (which incidentally corresponds to the surface having zero Gauss curvature at the point in question). Rephrased, we claim that the function $Q:[0,1] \rightarrow$ $\mathbb{R}$ defined by

$$
Q(a)=\int_{0}^{2 \pi}\left[2 a \cos 2 \varphi+1+a^{2}\right]^{\frac{1}{2}} d \varphi,
$$

takes its maximum value at $a=1$. This is verified by calculating that $Q^{\prime}(0)=0$ and $Q^{\prime \prime}(a) \geq 0$ for all $a \in[0,1]$ and deducing that $Q^{\prime}(a) \geq 0$ for all $a \in[0,1]$. Therefore throughout the interval $[0,1]$, we have $Q(a) \leq$ $Q(1)=8$. Exploiting this fact in (34) then yields

$$
h \leq 32 \sqrt{\left(1+\kappa_{1}^{2}\right)\left(1+\kappa_{2}^{2}\right)} \leq 16\left(1+\kappa_{1}^{2}+1+\kappa_{2}^{2}\right)=16\left(4+4 H^{2}-2 K\right),
$$

which we may integrate, using the Gauss-Bonnet formula again, to give

$$
\mu(\mathcal{M}) \leq 64 W .
$$

Equality may only be achieved here when $K \equiv 0$, and the principal curvatures are equal in magnitude. By retaining the function $Q$, instead of estimating it, or by not using the inequality $2 \sqrt{\left(1+\kappa_{1}^{2}\right)\left(1+\kappa_{2}^{2}\right)} \leq 4+4 H^{2}-2 K$, we would get a stronger estimate for $\mu(\mathcal{M})$, but a more complicated one.

We now require an exact expression for the total swept volume $\mu(\mathcal{M})$ in terms of the tangency map $\Phi$. Let us take an arbitrary point $(r, s) \in S^{2} \times S^{2}$ (analogous to the point $\xi$ in the proof of Theorem 3 ) and ask for which points $(a, b) \in S^{2} \times S^{2}$ does the torus $T_{a, b}$ contain the point $(r, s)$. A moment's thought reveals that $(r, s) \in T_{a, b}$ precisely when $(a, b) \in T_{r, s}$, so with $a$ and $b$ the components of $\sigma$ as above, the torus $T_{a, b}$ sweeps over $(r, s)$ at each point $p$ for which $\sigma(p) \in T_{r, s}$. We will shortly see that such points $p$ are characterised by the fact that the surface at $p$ is tangent to one of the tori in the foliation of tori $\mathbb{T}_{r}$ for which $\mathbb{T}_{0}$ lies in the plane $\alpha^{-1}(r, s)$. Therefore 
by definition of $\Phi$ there are precisely $\Phi\left(\alpha^{-1}(r, s)\right)$ points $p \in \mathcal{M}$ at which $\sigma(p) \in T_{r, s}$. By definition of $\mu$ we then see that

$$
\begin{aligned}
\mu(\mathcal{M}) & =\int_{S^{2} \times S^{2}} \Phi\left(\alpha^{-1}(y)\right) d y=16 \pi^{2} f_{S^{2} \times S^{2}} \Phi\left(\alpha^{-1}(y)\right) d y \\
& =16 \pi^{2} f_{G_{2,4}} \Phi
\end{aligned}
$$

since $S^{2} \times S^{2}$ has volume $16 \pi^{2}$. Let us now justify the characterisation of points $p \in \mathcal{M}$ with $\sigma(p) \in T_{r, s}$. We begin by choosing an orthonormal basis $\left\{e_{1}, \ldots, e_{4}\right\}$ of $\mathbb{R}^{4}$ so that $\alpha\left(e_{1} \wedge e_{2}\right)=(r, s)$. The preimage of the torus $T_{r, s}$ under $\alpha$ is then precisely the set of planes with no $e_{1} \wedge e_{2}$ or $e_{3} \wedge e_{4}$ components (since they are orthogonal in $\mathcal{P}$ to both $e_{1} \wedge e_{2}+e_{3} \wedge e_{4}$ and $\left.e_{1} \wedge e_{2}-e_{3} \wedge e_{4}\right)$. This set of planes may be written

$$
\Sigma=\left\{\left(\cos \theta e_{1}+\sin \theta e_{2}\right) \wedge\left(\cos \varphi e_{3}+\sin \varphi e_{4}\right) \mid \theta, \varphi \in[0,2 \pi)\right\} .
$$

Note that this can also be seen via the map $\gamma$; we know that $e_{1} \wedge e_{3}$ is in $\Sigma$, and since $T_{r, s}$ is the orbit of $\alpha\left(e_{1} \wedge e_{3}\right)$ under the subgroup of $S O(3) \times S O(3)$ which fixes $(r, s)$, the set $\Sigma$ must arise as the orbit of $e_{1} \wedge e_{3}$ under arbitrary rotations in the planes $e_{1} \wedge e_{2}$ and $e_{3} \wedge e_{4}$, which gives the set in (37) exactly. Now let us turn to the foliation of tori $\mathbb{T}_{r}$ for which $\mathbb{T}_{0}$ lies in the $e_{1} \wedge e_{2}$ plane (as used in the definition of $\Phi$ ). We parameterise $\mathbb{T}_{r}$ with $\theta, \varphi \in[0,2 \pi)$ according to

$$
u(\theta, \varphi)=r \cos \theta e_{1}+r \sin \theta e_{2}+\sqrt{1-r^{2}} \cos \varphi e_{3}+\sqrt{1-r^{2}} \sin \varphi e_{4},
$$

so that

$u^{*}(\theta, \varphi)=\sqrt{1-r^{2}} \cos \theta e_{1}+\sqrt{1-r^{2}} \sin \theta e_{2}-r \cos \varphi e_{3}-r \sin \varphi e_{4}$,

and thus

$$
u \wedge u^{*}=-\left(\cos \theta e_{1}+\sin \theta e_{2}\right) \wedge\left(\cos \varphi e_{3}+\sin \varphi e_{4}\right) .
$$

Therefore, the tangent planes to $\mathbb{T}_{r}$ (given by $*\left(u \wedge u^{*}\right)$ ) are elements of $\Sigma$. Conversely, we observe that any tangent plane at a point in $S^{3}$ will only lie in $\Sigma$ if it is tangent to the torus $\mathbb{T}_{r}$ passing through that point. The tangent planes at points on $\mathbb{T}_{0}$ and $\mathbb{T}_{1}$ are handled analogously, but separately. We conclude that $\sigma(p) \in T_{r, s}$ precisely at points $p$ where the surface $\mathcal{M}$ is tangent to a torus in the given foliation $\mathbb{T}_{r}$, as desired.

We may now combine (35) with (36) to give

$$
W \geq \frac{\mu(\mathcal{M})}{64}=\frac{\pi^{2}}{4} f_{G_{2,4}} \Phi,
$$

which concludes the proof of Theorem 4 . 
We end this section by sketching an alternative approach to proving Theorem 3 using the geometric constructions we have now introduced. The method involves integral geometry on $S O(3)$, the double cover of which is $S^{3}$. By making an identification between the two copies of $S^{2}$ in the target of $\sigma$, we can count the number of points on a subset $A \subset \mathcal{M}$ of the surface at which $a$ and $b$ (the components of $\sigma$ as before) map to the same point in the sphere $S^{2}$. More generally, given an element of $M \in S O(3)$, we can consider the number of points in $A$ at which $a$ agrees with the composed map $M \circ b$. Integrating this number over the whole of $S O(3)$ (with an invariant metric) provides a measure $\mu(A)$ of the subset $A$. Analogously to the proof we have detailed of Theorem 3, it turns out that we may bound the measure of the whole surface $\mathcal{M}$ from below using Morse theory on the function $f$ given in (25). We may then argue that for each point $(a(p), b(p))$ in the image of $\sigma$, there is a circle of rotations $M \in S O(3)$ for which $a(p)=M \circ b(p)$, and we may derive an expression (in terms of the mean and Gauss curvatures of $\mathcal{M}$ ) for the volume swept out by this circle in $S O(3)$ as we move the point $p$ on the surface. Since this volume is what the measure $\mu$ is measuring, we end up with a lower bound for a curvature integral. Pursuing these ideas a little further reveals that the method is essentially equivalent to the method of Sect. 4.1 and gives the same curvature estimate as in Theorem 3.

\subsection{Lower bounds for $\Phi$ - the proof of Corollary 5}

We now turn to the problem of establishing lower bounds for $\Phi$ using Morse theory. We have to work a little harder than previously to apply this theory since not all of the critical points of the Morse functions we construct have the geometric properties we require. Moreover, as we have already mentioned, it is inevitable that the bound for $\Phi$ will not be good enough to deduce the Willmore conjecture unless we use the conformal invariance of the problem, or appeal to special properties of the surface such as invariance under the antipodal map.

The following bound was claimed as we introduced $\Phi$ in Sect. 3 .

Lemma 2 For any immersed surface of genus $g$ in $S^{3}$, we have the pointwise estimate

$$
\Phi \geq 2(1+g),
$$

almost everywhere.

Proof. Given a plane $e_{1} \wedge e_{2}$, where $e_{1}$ and $e_{2}$ are orthogonal unit vectors in $\mathbb{R}^{4}$, we complete it to a positive orthonormal basis $\left\{e_{1}, \ldots, e_{4}\right\}$. To estimate $\Phi\left(e_{1} \wedge e_{2}\right)$ we consider, as a prototype Morse function, the function $f$ : $\mathcal{M} \rightarrow[0,1]$ defined by

$$
f(p)=F(u(p)),
$$


where $F: S^{3} \rightarrow[0,1]$ is defined on the ambient $\mathbb{R}^{4}$ by

$$
F(\mathbf{x})=\left\langle\mathbf{x}, e_{1}\right\rangle^{2}+\left\langle\mathbf{x}, e_{2}\right\rangle^{2} .
$$

We will be proving (38) for any plane $e_{1} \wedge e_{2}$ for which $f$ is indeed a Morse function (i.e. has no degenerate critical points) and the set of planes not satisfying this condition has measure zero. (At each point $p$ on the twodimensional surface $\mathcal{M}$, there is a two-dimensional set of planes for which $f$ has a critical point at $p$, but only a one-dimensional subset of these planes will make $f$ degenerate at $p$ - meanwhile, the complete set of planes is fourdimensional.) Note that the level sets $\left\{\mathbf{x} \in S^{3} \mid F(\mathbf{x})=r^{2}\right\}$ are precisely the tori in the family $\mathbb{T}_{r}$. Therefore, we see that the critical points of $f$ which have critical value in $(0,1)$ (i.e. neither 0 nor 1 ) are all points where the surface is tangent to one of the tori $\mathbb{T}_{r}$. If $f$ does not have 0 or 1 as a critical value, then Morse theory gives (38) immediately by providing $2(1+g)$ critical points for $f$ and hence $2(1+g)$ points of tangency with the foliation $\mathbb{T}_{r}$.

However, in general, the great circles $\mathcal{C}_{1}$ and $\mathcal{C}_{2}$ corresponding to $e_{1} \wedge e_{2}$ and $e_{3} \wedge e_{4}$ respectively, may intersect the surface $\mathcal{M}$ and there $f$ will take the value 0 or 1 . In that case, not all the $2(1+g)$ critical points of $f$ given to us by Morse theory need correspond to tangency points counted by $\Phi$; if one of the great circles has a tangential intersection with the surface then the intersection point is counted by $\Phi$, but if the intersection is transversal, then it is not counted. For the latter case, we are forced to use a little more Morse theory than the basic lower bound for the number of critical points.

The Betti numbers of $\mathcal{M}$ are $\beta_{0}=1, \beta_{1}=2 g$ and $\beta_{2}=1$. Let us denote by $c_{i}$ the number of critical points with index $i$, for $i=0,1,2$. The weak Morse inequalities (see [16]) then tell us that

$$
\begin{gathered}
c_{0} \geq \beta_{0}=1, \quad c_{1} \geq \beta_{1}=2 g, \quad c_{2} \geq \beta_{2}=1, \\
2-2 g=\beta_{0}-\beta_{1}+\beta_{2}=\chi(\mathcal{M})=c_{0}-c_{1}+c_{2} .
\end{gathered}
$$

In particular, it is (40) which gave us $c_{0}+c_{1}+c_{2} \geq 2(1+g)$. Note that each critical point of $f$ with critical value 0 is a minimum, and therefore has index zero, and is counted by $c_{0}$. Similarly each critical point of $f$ with critical value 1 is a maximum, and therefore has index two, and is counted by $c_{2}$. Meanwhile, all the critical points counted by $c_{1}$ have critical value in $(0,1)$, and hence correspond to points we seek where the surface $\mathcal{M}$ is tangent to one of the tori $\mathbb{T}_{r}$.

We wish to prove that there are at least $2(1+g)$ critical points of $f$ not corresponding to transversal intersections between $\mathcal{M}$ and either of the great circles $\mathcal{C}_{i}$. Suppose we have $n$ transversal intersections between $\mathcal{M}$ and $\mathcal{C}_{1}$, and $m$ transversal intersections between $\mathcal{M}$ and $\mathcal{C}_{2}$. If $n=m=0$ then all critical points of $f$ (of which there are at least $2(1+g)$ ) are counted 
by $\Phi$ and there is nothing more to check. If $m=0$ and $n>0$, then we must have $n \geq 2$ since $\mathcal{M}$ divides $S^{3}$ into (at least) two components. In this case $c_{0} \geq n$, and (41) and (40) imply

$$
c_{1}+c_{2}=c_{0}+2 c_{2}+2 g-2 \geq 2 g+n \geq 2(1+g) .
$$

Since $c_{1}$ and $c_{2}$ count critical points which are also counted by $\Phi$ (in this case) this is sufficient. The case $m>0$ and $n=0$ is essentially the same as the case $n>0$ and $m=0$. Finally, if both $n>0$ and $m>0$, then $c_{0} \geq n \geq 2$ and $c_{2} \geq m \geq 2$ and so (41) and (40) imply

$$
c_{1}=c_{0}+c_{2}+2 g-2=2 g+n+m-2 \geq 2(1+g) .
$$

Since $c_{1}$ always counts critical points which are counted by $\Phi$, the proof of the final case for $n$ and $m$ is complete.

Note that if $n>2$ or $m>2$ then we could get a better bound for $\Phi$ which could be used to show that surfaces which on average intersect great circles many times must have large Willmore energy.

An adaptation of the above argument gives an estimate for tori which is twice as strong, assuming that the surface has antipodal invariance.

Lemma 3 For any embedded torus in $S^{3}$ which is invariant under the antipodal map, we have the estimate

$$
\Phi \geq 8
$$

almost everywhere.

Corollary 5 clearly follows immediately from (5), Lemma 3 and Theorem 4.

Proof. Let us define $\tilde{\mathcal{M}}$ to be the quotient of the surface $\mathcal{M}$ by the equivalence relation which identifies the pairs of points mapped to antipodal points in $S^{3}$ by $u$. We denote by $\tilde{u}: \tilde{\mathcal{M}} \rightarrow \mathbb{R} P^{3}$ the map induced by $u$. The Euler characteristic of $\tilde{\mathcal{M}}$, being half that of $\mathcal{M}$, is zero. Moreover, $\tilde{\mathcal{M}}$ cannot be nonorientable since that would make $\tilde{u}$ an embedding of the Klein bottle into $\mathbb{R} P^{3}$ which is impossible [20]. Therefore, $\tilde{\mathcal{M}}$ is also a torus.

Now on $\tilde{\mathcal{M}}$, the function $f$ defined in (39) is well-defined, and we may argue as above that for almost all planes $e_{1} \wedge e_{2}$, it is a Morse function and we may find at least $2(1+g)=4$ critical points corresponding to points on the quotient surface in $\mathbb{R} P^{3}$ (given by $\tilde{u}$ ) where the surface is tangent to a torus in the foliation $\mathbb{T}_{r}$ (which may be seen, after we have taken the quotient by the antipodal map, in $\mathbb{R} P^{3}$ ). It remains to lift these four (or more) points in $\tilde{\mathcal{M}}$ to the corresponding eight (or more) points in $\mathcal{M}$, and at these points the surface will still be tangent to tori in $\mathbb{T}_{r}$ and will all be counted by $\Phi$. 
We end this section by sketching why, with the hindsight of the calculations in Sect. 4.2, we have $\Phi \equiv 2$ almost everywhere when the surface is a round 2 -sphere embedded in $S^{3}$, and $\Phi \equiv 8$ almost everywhere when the surface is the Clifford torus. As we saw in Sect. 4.2, the quantity $\Phi\left(\alpha^{-1}(r, s)\right)$ turns out to count precisely the number of points in $\mathcal{M}$ which are mapped to the torus $T_{r, s}$ by $\sigma$.

When $\mathcal{M}$ is a 2 -sphere embedded as a round sphere in $S^{3}$, by making an appropriate identification of the two copies of $S^{2}$ in the target $S^{2} \times S^{2}$ of $\sigma$ (which is equivalent to picking an appropriate basis $\left\{e_{1}, \ldots, e_{4}\right\}$ for the ambient $\mathbb{R}^{4}$ if we are using the bases in (26) and (27)) we may assume that both components $a$ and $b$ of $\sigma$ are identical - both being essentially the identity map, up to a uniform expansion depending on the size of the original embedded 2-sphere. In this case, $\Phi\left(\alpha^{-1}(r, s)\right)$ counts the points on $S^{2}$ which are orthogonal to both $r$ and $s$; if $r \neq s$ and $r \neq-s$, then $\Phi\left(\alpha^{-1}(r, s)\right)=2$, whilst for the remaining two 2-spheres of oriented planes $\left\{\alpha^{-1}(r, s) \mid r= \pm s\right\}$, the value of $\Phi$ is infinity.

For the Clifford torus, an explicit calculation of the components $a$ and $b$ of $\sigma$ has been given in (31) and (32). In the coordinates used there, the planes $e_{1} \wedge e_{2},-e_{1} \wedge e_{2}, e_{3} \wedge e_{4}$ and $-e_{3} \wedge e_{4}$, correspond to $(r, s)=$ $(0,0, \pm 1,0,0, \pm 1)$, and for these, $\Phi$ is infinity. For all other planes $\alpha^{-1}(r, s)$, the torus $T_{r, s}$ intersects the image of $\sigma$ twice at each of four points and so $\Phi$ takes the value eight for these planes. For example, if $r=s=(1,0,0)$, then $\sigma$ maps into $T_{r, s}$ at points $(x, y)$ where $\cos \sqrt{2}(x+y)= \pm 1$ and $\cos \sqrt{2}(x-y)= \pm 1$, or equivalently $\sqrt{2}(x+y)=0$ and $\sqrt{2}(x-y)=0$ modulo $\pi$. These are the eight points

$$
\begin{gathered}
\sqrt{2}(x, y) \in\left\{(0,0),\left(\frac{\pi}{2}, \frac{\pi}{2}\right),(\pi, 0),(0, \pi),(\pi, \pi),\right. \\
\left.\left(\frac{3 \pi}{2}, \frac{\pi}{2}\right),\left(\frac{\pi}{2}, \frac{3 \pi}{2}\right),\left(\frac{3 \pi}{2}, \frac{3 \pi}{2}\right)\right\} .
\end{gathered}
$$

In particular $\Phi \equiv 8$ almost everywhere, which shows Lemma 3 to be sharp.

\subsection{Homotopically nontrivial Gauss maps for tori - the proof of Theorem 6}

The strategy of the proof of Theorem 6, as sketched in Sect. 3, will be to exploit the fact that the surface $\mathcal{M}$ is a torus to construct an extended Gauss map which will lie in a nontrivial homotopy class, and whose harmonic energy we will be able to bound from below as a result. This will then give us sharp bounds on the Willmore energy, with an error term depending on the least energy of vector fields on the surface.

Let us fix some positive basis $\left\{e_{1}, \ldots, e_{4}\right\}$ of $\mathbb{R}^{4}$. Given a vector field $v \in \Sigma$, where $\Sigma$ was defined in Theorem 6 , we define $w \in \Sigma$ to be the field 
obtained by rotating $v$ by an angle of $\frac{\pi}{2}$. In other words, if

$$
v=\frac{1}{\left|\frac{\partial u}{\partial x}\right|}\left(\cos \theta \frac{\partial u}{\partial x}+\sin \theta \frac{\partial u}{\partial y}\right),
$$

for some $\theta: \mathcal{M} \rightarrow \mathbb{R}$, then

$$
w=\frac{1}{\left|\frac{\partial u}{\partial x}\right|}\left(-\sin \theta \frac{\partial u}{\partial x}+\cos \theta \frac{\partial u}{\partial y}\right),
$$

so that $\{v, w\}$ form a positive orthonormal basis for the tangent space with the standard orientation induced from $\mathbb{R}^{2}$. (Let us recall that $u$ is conformal.)

Given $v$, and hence $w$, we define the extended Gauss map

$$
\Upsilon: \mathcal{M} \rightarrow S O(4)
$$

to be the rotation in $\mathbb{R}^{4}$ which sends $\left\{e_{1}, \ldots, e_{4}\right\}$ to $\left\{u, u^{*}, v, w\right\}$. Note that $\Upsilon$ depends not only on the torus in $S^{3}$ but also on the unit vector field $v$.

Let us calculate $\Upsilon$ for the immersion $u_{0}$ given in (16) as

$$
u_{0}(x, y)=\frac{1}{\sqrt{2}}\left(\cos 2 \pi x, \sin 2 \pi x, \cos \frac{2 \pi y}{d}, \sin \frac{2 \pi y}{d}\right),
$$

when we take $v$ to be

$$
v(x, y)=\frac{1}{\sqrt{2} \pi} \frac{\partial u}{\partial x}(x, y)=(-\sin 2 \pi x, \cos 2 \pi x, 0,0) .
$$

Since

$$
u_{0}^{*}(x, y)=\frac{1}{\sqrt{2}}\left(\cos 2 \pi x, \sin 2 \pi x,-\cos \frac{2 \pi y}{d},-\sin \frac{2 \pi y}{d}\right),
$$

and

$$
w(x, y)=\left(0,0,-\sin \frac{2 \pi y}{d}, \cos \frac{2 \pi y}{d}\right),
$$

we may simply write down the matrix $\Upsilon(x, y)$. However, we prefer to see $\Upsilon(x, y)$ geometrically as the fixed rotation $\Upsilon(0,0)$ followed by a simultaneous rotation of $2 \pi x$ in the $e_{1} \wedge e_{2}$ plane and $\frac{2 \pi y}{d}$ in the $e_{3} \wedge e_{4}$ plane.

In fact, we will be dealing mainly with the map $\Psi: \mathcal{M} \rightarrow S O(3)$ defined to be the composition $p_{1} \circ \gamma \circ \Upsilon$ of $\Upsilon: \mathcal{M} \rightarrow S O(4), \gamma: S O(4) \rightarrow S O(3) \times$ $S O(3)$ as defined in Sect. 4.2, and the projection $p_{1}: S O(3) \times S O(3) \rightarrow$ $S O(3)$ onto the first copy of $S O(3)$. The group $S O(3)$ is topologically $\mathbb{R} P^{3}$, and a loop $\varsigma$ in $S O(3)$ corresponding to a full rotation of $2 \pi$ about a fixed axis is not contractible. As before, we endow the target $S O(3)$ of $\Psi$ with an invariant metric, but now we make it unique by fixing the normalisation so that the length of a shortest noncontractible loop $\varsigma$ is $2 \pi$. (Note that the 
normalisation which makes this length equal to $\pi$ would also be natural since it would make the universal cover of $S O(3)$ isometric to $S^{3}$.)

Given our geometric explanation of $\Upsilon$ for the immersion $u_{0}$, and our geometric understanding of the map $\gamma: S O(4) \rightarrow S O(3) \times S O(3)$ from Sect. 4.2, we see that for $u_{0}$, the element $\Psi(x, y) \in S O(3)$ is some fixed rotation (corresponding to $\Upsilon(0,0)$ ) followed by a rotation about a fixed axis of $2 \pi x+\frac{2 \pi y}{d}$.

Let us denote by $\eta_{1}:[0,1] \rightarrow \mathcal{M}$ and $\eta_{2}:[0, d] \rightarrow \mathcal{M}$ the nontrivial closed paths defined by

$$
\eta_{1}(t)=(t, 0) \quad \text { and } \quad \eta_{2}(t)=(0, t) .
$$

When $u=u_{0}$, our analysis of $\Psi$ above tells us that $\Psi \circ \eta_{1}$ is a nontrivial loop in $S O(3)$ - indeed $\Psi \circ \eta_{1}(t)$ is the composition of a fixed rotation and a rotation about a fixed axis of $2 \pi t$. Similarly, $\Psi \circ \eta_{2}$ is a nontrivial loop in $S O(3)$. We have assumed that the more general $u$ considered in Theorem 6 may be deformed to $u_{0}$ through a family of immersions, and hence $\Psi \circ \eta_{1}$ and $\Psi \circ \eta_{2}$ remain topologically nontrivial loops in $S O(3)$ for the more general $u$. Moreover, we have that for any $\eta \in\left[\eta_{1}\right] \in \pi_{1}(\mathcal{M})$ or $\eta \in\left[\eta_{2}\right] \in \pi_{1}(\mathcal{M}), \Psi \circ \eta$ is nontrivial and therefore must have length at least $2 \pi$, with our normalisation. We may use this fact to control the harmonic energy $E(\Psi)$ of $\Psi$ from below, as we now describe. For each $s \in[0, d]$, we consider the path $\eta_{1}^{s}(t)=(t, s)$. Since $\Psi \circ \eta_{1}^{s}$ has length at least $2 \pi$, we may estimate

$$
2 \pi \leq \int_{\eta_{1}^{s}}\left|\Psi_{*}\left(\frac{\partial}{\partial x}\right)\right| \leq\left(\int_{\eta_{1}^{s}}\left|\Psi_{*}\left(\frac{\partial}{\partial x}\right)\right|^{2}\right)^{\frac{1}{2}} .
$$

Squaring, and integrating with respect to $s$, yields

$$
4 \pi^{2} d \leq \int_{\mathcal{M}}\left|\Psi_{*}\left(\frac{\partial}{\partial x}\right)\right|^{2} .
$$

Repeating this argument for paths $\eta_{2}^{s}:[0, d] \rightarrow \mathcal{M}$ given by $\eta_{2}^{s}(t)=(s, t)$ in the perpendicular direction (with $s \in[0,1]$ ) gives

$$
2 \pi \leq \int_{\eta_{2}^{s}}\left|\Psi_{*}\left(\frac{\partial}{\partial y}\right)\right| \leq d^{\frac{1}{2}}\left(\int_{\eta_{2}^{s}}\left|\Psi_{*}\left(\frac{\partial}{\partial y}\right)\right|^{2}\right)^{\frac{1}{2}},
$$

and hence

$$
\frac{4 \pi^{2}}{d} \leq \int_{\mathcal{M}}\left|\Psi_{*}\left(\frac{\partial}{\partial y}\right)\right|^{2} .
$$

The combination of (42) and (43) now gives the required estimate

$$
E(\Psi)=\frac{1}{2} \int_{\mathcal{M}}\left(\left|\Psi_{*}\left(\frac{\partial}{\partial x}\right)\right|^{2}+\left|\Psi_{*}\left(\frac{\partial}{\partial y}\right)\right|^{2}\right) \geq 2 \pi^{2}\left(d+\frac{1}{d}\right) .
$$


The next task is to calculate $E(\Psi)$ in terms of the Willmore energy and $\mathcal{E}(v)$. Given a point $p$ in $\mathcal{M}$, let $f_{1}$ be the unit vector corresponding to the first principal curvature direction (or any unit tangent vector if $p$ is umbilic). We would like to know the length of $\Psi_{*}\left(f_{1}\right)$ in $T_{p} S O(3)$. By the invariance properties of the metric on $S O(3)$, we may assume that $e_{1}=u(p), e_{2}=$ $u^{*}(p), e_{3}=v(p)$ and $e_{4}=w(p)$. Moreover, we may assume that $u_{*}\left(f_{1}\right)=v$ at $p$ by rotating $v$ (and $w$ ) in the tangent space by a uniform amount (over the whole of $\mathcal{M})$ which affects neither the energy $\mathcal{E}(v)$ of $v$ nor the length of $\Psi_{*}\left(f_{1}\right)$. (This rotation corresponds to adding a fixed amount to $\theta$.) With these conventions, $\Upsilon(p)$ is simply the identity, and defining $\omega_{1}: \mathcal{M} \rightarrow \mathbb{R}$ by

$$
\omega_{1}:=\left\langle v_{*}\left(f_{1}\right), w\right\rangle=-\left\langle w_{*}\left(f_{1}\right), v\right\rangle,
$$

we have

$$
\begin{gathered}
u_{*}\left(f_{1}\right)=(0,0,1,0), \quad\left(u^{*}\right)_{*}\left(f_{1}\right)=\left(0,0, \kappa_{1}, 0\right), \\
v_{*}\left(f_{1}\right)=\left(-1,-\kappa_{1}, 0, \omega_{1}\right), \quad w_{*}\left(f_{1}\right)=\left(0,0,-\omega_{1}, 0\right) .
\end{gathered}
$$

Therefore, the derivative of $\Upsilon$ in the $f_{1}$ direction is given by

$$
\Upsilon_{*}\left(f_{1}\right)=\left(\begin{array}{cccc}
0 & 0 & -1 & 0 \\
0 & 0 & -\kappa_{1} & 0 \\
1 & \kappa_{1} & 0 & -\omega_{1} \\
0 & 0 & \omega_{1} & 0
\end{array}\right)
$$

We may now calculate the eigenvalues of this matrix to be $0,0, \pm i$ $\sqrt{1+\kappa_{1}^{2}+\omega_{1}^{2}}$. In other words, $\Upsilon_{*}\left(f_{1}\right)$ is the generator for rotation which fixes one plane in $\mathbb{R}^{4}$, and moves the other at a 'rate' $\sqrt{1+\kappa_{1}^{2}+\omega_{1}^{2}}$. More concretely, $\Upsilon_{*}\left(f_{1}\right)$ with respect to a different orthonormal basis would be

$$
\left(\begin{array}{cccc}
0 & 0 & 0 & 0 \\
0 & 0 & 0 & 0 \\
0 & 0 & 0 & -\sqrt{1+\kappa_{1}^{2}+\omega_{1}^{2}} \\
0 & 0 & \sqrt{1+\kappa_{1}^{2}+\omega_{1}^{2}} & 0
\end{array}\right) .
$$

Using our understanding of the map $\gamma: S O(4) \rightarrow S O(3) \times S O(3)$, we then see that $\Psi_{*}\left(f_{1}\right)$ generates a rotation at 'rate' $\sqrt{1+\kappa_{1}^{2}+\omega_{1}^{2}}$ in the sense that with respect to a certain orthonormal basis,

$$
\Psi_{*}\left(f_{1}\right)=\left(\begin{array}{ccc}
1 & 0 & 0 \\
0 & 0 & -\sqrt{1+\kappa_{1}^{2}+\omega_{1}^{2}} \\
0 & \sqrt{1+\kappa_{1}^{2}+\omega_{1}^{2}} & 0
\end{array}\right) .
$$

We may conclude, with the normalisation for $S O(3)$ we have chosen, that

$$
\left|\Psi_{*}\left(f_{1}\right)\right|^{2}=1+\kappa_{1}^{2}+\omega_{1}^{2} .
$$


Similarly, if $f_{2}$ is taken to be in the second principal curvature direction, then

$$
\left|\Psi_{*}\left(f_{2}\right)\right|^{2}=1+\kappa_{2}^{2}+\omega_{2}^{2},
$$

where $\omega_{2}: \mathcal{M} \rightarrow \mathbb{R}$ is now given by $\omega_{2}:=\left\langle v_{*}\left(f_{2}\right), w\right\rangle=-\left\langle w_{*}\left(f_{2}\right), v\right\rangle$. We therefore have the harmonic energy of $\Psi$ given by

$$
\begin{aligned}
E(\Psi) & =\frac{1}{2} \int_{\mathcal{M}}|\nabla \Psi|^{2}=\frac{1}{2} \int_{\mathcal{M}}\left(1+\kappa_{1}^{2}+\omega_{1}^{2}\right)+\left(1+\kappa_{2}^{2}+\omega_{2}^{2}\right) \\
& =\int_{\mathcal{M}}\left(2+2 H^{2}-K\right)+\frac{1}{2} \int_{\mathcal{M}}\left(\omega_{1}^{2}+\omega_{2}^{2}\right) \\
& =2 W+\mathcal{E}(v) .
\end{aligned}
$$

It remains to combine this expression with the bound in (44) to conclude

$$
W+\frac{1}{2} \mathcal{E}(v) \geq \pi^{2}\left(d+\frac{1}{d}\right) .
$$

Remark 2 The technology and ideas used in the proofs of Theorems 4 and 6 may be applied to the slightly different problem of proving (3) for Lagrangian tori in $\mathbb{R}^{4}$ (see [17]). A moment's thought reveals that the Lagrangian condition is equivalent to requiring the map $\sigma: \mathcal{M} \rightarrow S^{2} \times S^{2}$ from Sect. 4.2 (which still makes sense for surfaces in $\mathbb{R}^{4}$ ) to map into a great circle in one of the 2-spheres in the target. The crucial point now is that the Gauss map lies in a nontrivial homotopy class, described by the Maslov class, and we can therefore pursue the strategy of Sect. 4.4 to get a lower bound for the harmonic energy of the Gauss map (and hence for the Willmore energy) in terms of the conformal class of the torus, and the Maslov class.

Incidentally, for surfaces in $S^{3}$, we saw in Sect. 4.2 that the Gauss curvature of the surface is equal to the Jacobian of the components $a$ and $b$ of $\sigma$; for Lagrangian tori taking values in $S^{3}$, one of these components maps into a great circle in its target $S^{2}$ and therefore the surface must be flat, at which point we may apply Theorem 1 .

Acknowledgements. I would like to thank the numerous mathematicians who have shown their enthusiasm for this project, and shared the results of their own exploits. In particular, I would like to thank Nikolai Nadirashvili, with whom I discussed the Willmore conjecture on many occasions. This work was undertaken at the ETH - Zürich, supported by the Royal Society's European Science Exchange Programme, and at the IHES, supported by the European Postdoctoral Institute. I would like to thank these institutions for their hospitality and support. 


\section{References}

1. M. Babich, A. Bobenko, Willmore tori with umbilic lines and minimal surfaces in hyperbolic space. Duke Math. J. 72 (1993) 151-185

2. T. F. Banchoff, The spherical two-piece property and tight surfaces in spheres. J. Differential Geometry 4 (1970) 193-205

3. M. Berger, P. Gauduchon, E. Mazet, 'Le spectre d'une variété riemannienne.' SLN 194. Springer 1971

4. W. Blaschke, 'Vorlesungen über Differentialgeometrie, III.' Springer 1929

5. M. Cai, On functionals of Willmore type. Preprint.

6. T. E. Cecil, P. J. Ryan, 'Tight and taut immersions of manifolds.' Pitman 1985

7. B. Y. Chen, On the total curvature of immersed manifolds V. C-surfaces in Euclidean m-space. Bull. Inst. Math., Acad. Sin. 9 (1981) 509-516

8. S. S. Chern, R. K. Lashof, On the total curvature of immersed manifolds. I, II. Amer. J. Math. 79 (1957) 306-318; Michigan math J. 5 (1958) 5-12

9. M. L. Curtis, 'Matrix groups.' Springer-Verlag 1979

10. J. Eells, L. Lemaire, A report on harmonic maps. Bull. London Math. Soc. 10 (1978) $1-68$

11. P. Li, S.-T. Yau, A new conformal invariant and its applications to the Willmore conjecture and the first eigenvalue of compact surfaces. Invent. Math. 69 (1982) 269291

12. R. Kusner, Comparison surfaces for the Willmore problem. Pac. J. Math. 138 (1989) 317-345

13. R. Kusner, Estimates for the biharmonic energy on unbounded planar domains, and the existence of surfaces of every genus that minimize the squared-mean-curvature integral. 'Elliptic and parabolic methods in geometry.' B. Chow et al ed., Wellesley (1996) 67-72

14. R. Langevin, H. Rosenberg, On curvature integrals and knots. Topology 15 (1976) 405-416

15. H. B. Lawson, Complete minimal surfaces in $S^{3}$. Ann. Math. 92 (1970) 335-374

16. J. W. Milnor, 'Morse theory.' Ann. Math. Stud. 51. Princeton University Press 1963

17. W. P. Minicozzi, The Willmore functional on Lagrangian tori: Its relation to area and existence of smooth minimizers. J. Am. Math. Soc. 8 (1995) 761-791

18. S. Montiel, A. Ros, Minimal immersions of surfaces by the first eigenfunctions and conformal area. Invent. Math. 83 (1986) 153-166

19. U. Pinkall, Hopf tori in $S^{3}$. Invent. Math. 81 (1985) 379-386

20. A. Ros, The Willmore conjecture in the real projective space. Preprint 1997

21. L. M. Simon, Existence of surfaces minimizing the Willmore functional. Comm. Anal. Geom. 1 No.2 (1993) 281-326

22. G. Thomsen, Über Konforme Geometrie, I: Grundlagen der Konformen Fläschentheorie. Abh. Math. Sem. Hamburg 3 (1923) 31-56

23. P. M. Topping, The harmonic map heat flow from surfaces. PhD Thesis, University of Warwick 1996

24. P. M. Topping, Mean Curvature Flow and Geometric Inequalities. J. Reine Angew. Math. 503 (1998) 47-61

25. T. J. Willmore, 'Riemannian geometry.' Oxford University Press 1993

26. S.-T. Yau, Problem section. 'Seminar on differential geometry.' Ann. Math. Stud. 102. Princeton University Press (1982) 669-706 